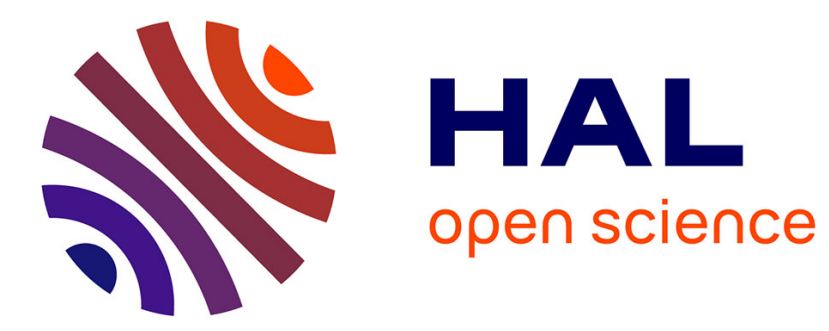

\title{
On the present shape of the Oort cloud and the flux of "new" comets
}

\author{
M. Fouchard, H. Rickman, Ch. Froeschlé, G.B. Valsecchi
}

\section{To cite this version:}

M. Fouchard, H. Rickman, Ch. Froeschlé, G.B. Valsecchi. On the present shape of the Oort cloud and the flux of "new" comets. Icarus, 2017, 292, pp.218-233. 10.1016/j.icarus.2017.01.013 . hal-01525926

\section{HAL Id: hal-01525926 \\ https://hal.sorbonne-universite.fr/hal-01525926}

Submitted on 22 May 2017

HAL is a multi-disciplinary open access archive for the deposit and dissemination of scientific research documents, whether they are published or not. The documents may come from teaching and research institutions in France or abroad, or from public or private research centers.
L'archive ouverte pluridisciplinaire HAL, est destinée au dépôt et à la diffusion de documents scientifiques de niveau recherche, publiés ou non, émanant des établissements d'enseignement et de recherche français ou étrangers, des laboratoires publics ou privés. 
Long term evolution of an initial set of $10^{7}$ Oort cloud comets is performed for the age of the solar system taking into account the action of passing stars using 10 different sequences of stellar encounters, Galactic tides and the gravity of the giant planets. The initial conditions refer to a disk-shaped Oort cloud precursor, concentrated toward the ecliptic with perihelia in the region of Uranus and Neptune. Our results show that the shape of the Oort cloud quickly reach a kind of steady state beyond a semi-major axis greater than about $2000 \mathrm{AU}$ (this threshold depending on the evolution time-span), with a Boltzmann distribution of the orbital energy. The stars act in an opposite way to what was found in previous papers, that is they emptied an initial Tidal Active Zone that is overfilled with respect to the isotropic case. Consequently, the inclusion of stellar perturbations strongly affect the shape of the Oort spike. On the contrary, the Oort spike shape appears to be poorly dependent on the stellar sequences used, whereas the total flux of observable comets and the proportion of retrograde comets for the inner part of the spike are significantly dependent of it. Then it has been highlighted that the total flux, the shape of the Oort spike and the shape of the final Oort cloud are almost independent of the initial distribution of orbital energy considered.

\section{Introduction}

Recent years have seen a lot of research activities devoted to the Oort cloud. Some of these have focused on the origin of the cloud by simulating the increase of perihelion distances of scattered disk objects and the consequent decoupling from the gravitational influence of the planets. Others have started from a cloud

\footnotetext{
* Corresponding author

Email addresses: fouchard@imcce.fr (M. Fouchard), hans@fysast.uu.se (H. Rickman), froesch@obs-nice.fr (Ch. Froeschlé), giovanni@iaps.inaf.it (G.B. Valsecchi)
} 
more or less resembling the present one and followed its long term evolution and the flux and distribution of new comets currently arising from it.

In the first category of papers, some consider Oort cloud formation during the earliest stages of the solar system while modeling different scenarios for the dynamics (e.g. Brasser et al., 2006, 2007; Kaib and Quinn, 2008; Leto et al., 2008; Paulech et al., 2010; Levison et al., 2010). Others are less specific about the timing while concentrating more on the location in the Galaxy, where the solar system was situated (Brasser et al., 2010; Kaib et al., 2011), and finally, the work of Brasser and Morbidelli (2013) assumed that both the scattered disk and the Oort cloud were formed as a consequence of giant planet migration within the Nice Model. The latter may have taken place at the time of the Late Heavy Bombardment about 4.1 Gyr ago (Morbidelli et al., 2012). Alternatively, if the Late Heavy Bombardment has a different explanation, this migration - and the birth of the Oort cloud - might have occurred at a much earlier time.

In the second category, some papers have explored the interplay of stellar encounters and Galactic tides in providing new comets from the cloud (Rickman et al., 2008; Fouchard et al., 2011a,b), while others have drawn attention to the role of planetary perturbations in activating the inner core of the cloud and bringing comets from this region into the reach of stars or Galactic tides (Kaib and Quinn, 2009; Fouchard et al., 2013).

From all these results, we may conclude that the inner core of the Oort cloud has the potential to be quite important in determining the rate of arrival of new comets as well as their ecliptic inclination distribution. Moreover, its structure (degree of flattening toward the ecliptic) and population size may differ, depending on the circumstances under which the Oort cloud was formed. One particular issue in this context is the degree of isotropy of the current cloud and the inclination distribution of new comets. Fouchard et al. (2013) found that most of the new comets are so-called creepers, meaning that they did not jump across the Jupiter-Saturn barrier at once before discovery, and such comets showed a tendency to prefer retrograde orbits. Thus, if the Oort cloud is indeed isotropic, new comets should be mostly retrograde - in apparent contradiction to the commonly held view that the new comets show no such tendency. Kaib and Quinn (2009) had come to a similar conclusion and found this to hold even assuming a flattened inner core.

Resolving this issue is one of several tasks that may be tackled by a new generation of long term Oort cloud models, including both the formation and evolution of the cloud until the present time. In the present paper, we begin to perform such simulations. As will be shown, the results are encouraging. We find that tracing the evolution of the Oort cloud all the way from an initial prograde, flattened disk until the arrival of the current new comets indeed changes the picture in some important respects. Even so, the model we employ does not account for the influence of the solar birth cluster, solar migration in the Galaxy or GMC 
encounters, and this points to the importance of further, more elaborate models of the kind we are now introducing. It is fair to say that, by neglecting the birth cluster, we model an Oort Cloud such as the one formed as a result of a planetary migration within the Nice Model (Brasser and Morbidelli, 2013).

In Sect. 2 we present our initial conditions for the Oort cloud and the dynamical model of our simulations. Section 3 shows the results concerning the shape of the cloud in terms of distributions of semi-major axis, inclination, and the population of the Tidally Active Zone (Fouchard et al., 2011a). Section 4 concentrates on the observable Oort spike and its distribution of inclinations, and in Sect. 5 we study the influence of the initial energy distribution of the cloud on the present delivery of new comets. Section 6 is devoted to a discussion on the definition of the Oort cloud and its steady state and a summary of our conclusions is presented in Sect. 7.

\section{Initial conditions and simulations}

To simulate the precursor of the Oort cloud in the form of a scattered disk, a random initial set of $10^{7}$ comets is constructed using uniform distributions of the following elements: the orbital energy is such that the semi-major axis $(a)$ is between $1100 \mathrm{AU}$ and $50000 \mathrm{AU}$, the perihelion distance $(q)$ is between 15 and $32 \mathrm{AU}$, the inclination $(i)$ is between $0^{\circ}$ and $20^{\circ}$, and the three last parameters (argument of perihelion, longitude of the ascending node and mean anomaly) are between $0^{\circ}$ and $360^{\circ}$. All the angles are given with respect to the ecliptic plane. The flat distribution of the initial orbital energy is not considered as real. It will be used in Sec. 5 as a basis for modeling different, more realistic distributions.

This set of initial orbital elements represents a scattered disk formed by the gravitational perturbations of Uranus and Neptune (Duncan et al., 1987; Dones et al., 2004). During such a process, the argument of perihelion may be clustered near $180^{\circ}$ and $0^{\circ}$ (Brasser, private communication), but such accumulation is neglected in the present study.

The scattering time scale due to Uranus and Neptune may be estimated at roughly 200 Myr (Brasser and Morbidelli, 2013). Therefore, we choose the injection time of a comet into the disk randomly with an uniform distribution between 0 and $T_{G}$, where $T_{G}$ is the period of revolution of the Sun around the Galactic centre. Currently, the value of $T_{G}$ is rather close to $200 \mathrm{Myr}$ (see below), and we do not allow for the possibility of previous radial migration of the solar system in the Galaxy. This period is used because the time of injection has an influence on the position of the Galactic centre with respect to the ecliptic plane, and consequently on the action of the radial Galactic tide (Levison et al., 2006). Using an uniform distribution of the injection time between 0 and $T_{G}$ ensures that no position of the Sun with respect to the Galactic center is favoured. 
We assume time $t=0$ to be an integer multiple of $T_{G}$ away from the present time. Thus, when $t=k \cdot T_{G}$, with $k \in \mathbb{N}$, the position of the Galactic center corresponds to the present one. According to the parameters used for the Galactic tides (Levison et al., 2001), we have $T_{G} \approx 236 \mathrm{Myr}$.

After being injected into the cloud each comet evolves in a model including Galactic tides, passing stars and planetary perturbations (Fouchard et al., 2014a) ${ }^{1}$. The evolution stops either when the comet reaches a heliocentric distance larger than $4 \times 10^{5} \mathrm{AU}$, when it reaches a semi-major axis smaller than $100 \mathrm{AU}$ or, finally, when it impacts the Sun or a planet. The full set of parameters of each comet remaining inside the cloud is stored when $t=k \cdot T_{G}-\Delta_{R}$ where $k=17, \ldots, 21$, corresponding to $t \approx 4.0$ Gyr to $t \approx 4.9$ Gyr and $\Delta_{R}=30$ Myr. Five final snapshots of the remaining comets are thus obtained.

After each snapshot, all the remaining comets evolve for a time span equal to $\Delta_{R}$ - a "relaxing" period, during which all stars that may produce moderate to strong comet showers are removed immediately. Specifically, the stars that may inject more than 10 comets into the observable region (heliocentric distance less than $5 \mathrm{AU}$ ) according to the power laws given by Eqs. (1,2) below (Fouchard et al., 2011b) are removed from the stellar sequence.

Thereafter, each comet evolves further until its next perihelion passage, still without any showerproducing stars. For a comet to be counted as observable, it must pass within 5 AU of the Sun at this perihelion passage. Because of the relaxing period, we are sure that the direct effects of significant comet showers have been removed, which is consistent with what is currently observed (Wiegert and Tremaine, 1999). In addition, when a comet is observable, the position of the Galactic centre, i.e. the influence of the radial Galactic tide, is similar to the present one (in Levison et al., 2006, a relation between the position of the Galactic center and the Oort cloud dynamics is highlighted).

Ten simulations were made using ten different sequences of stellar encounters. These will be referred to by the number of the sequence used. In addition, and for comparison only, one simulation was made without stellar perturbations, i.e., using only the Galactic tides and the planetary perturbations. This simulation is referred to as the TP-model.

Let us now describe the differences between the ten stellar sequences used. For this we consider only the stars passing before $t=17 T_{G}-\Delta_{R}$, i.e. the first storage time. In order to evaluate the characteristics of a stellar sequence, we have used the tool developed in Fouchard et al. (2011b). That is, for a fully thermalized Oort cloud of a million of comets with a uniform distribution of the orbital energy for semi-major axes between 3000 and $50000 \mathrm{AU}$, one is able to estimate the number $N_{\star}$ of comets directly injected into the

\footnotetext{
${ }^{1}$ The model of planetary perturbations is such that a perturbation is computed each time a comet passes at less than 60 AU from the Sun, using the part of the trajectory at less than 150 AU. These two parameters are slightly different from those in Fouchard et al. (2014a)
} 


\begin{tabular}{c|r|ccc} 
seq. & $\Sigma_{N_{\star}>10} N_{\star}$ & \multicolumn{4}{|c}{ Num. of stars with $N_{\star}>$} \\
$\#$ & & 10 & 100 & 500 \\
\hline 1 & 4001.1 & 142 & 3 & 0 \\
2 & 4612.4 & 123 & 10 & 0 \\
3 & 8127.5 & 139 & 14 & 4 \\
4 & 12549.9 & 129 & 11 & 3 \\
5 & 3917.6 & 132 & 3 & 0 \\
6 & 4543.4 & 133 & 8 & 0 \\
7 & 13490.3 & 140 & 8 & 4 \\
8 & 3562.8 & 128 & 5 & 0 \\
9 & 4538.7 & 138 & 5 & 1 \\
10 & 4044.9 & 130 & 7 & 0
\end{tabular}

Table 1: For each stellar sequence are given, from left to right, the number of the stellar sequence, the total estimated number of comets directly observable by stars with $N_{\star}>10$, and the numbers of stars able to directly inject more than 10 , 100 and 500 comets into the observable region. See the main text for how these numbers are computed.

observable region by a single star using the following power laws, according to the value of $M_{\star} / V_{\star}$ (where $M_{\star}$ is the stellar mass and $V_{\star}$ is the stellar speed at infinity):

$$
N_{\star}=16.23\left(\frac{M_{\star}}{M_{\odot}}\right)^{1.82}\left(\frac{40 \mathrm{~km} / \mathrm{s}}{V_{\star}}\right)^{1.82}\left(\frac{20000 \mathrm{AU}}{d_{\odot}}\right)^{0.91}
$$

if $M_{\star} / M_{\odot} \cdot 40 \mathrm{~km} \cdot \mathrm{s}^{-1} / V_{\star}<0.60077$, and

$$
N_{\star}=12.83\left(\frac{M_{\star}}{M_{\odot}}\right)^{0.89}\left(\frac{40 \mathrm{~km} / \mathrm{s}}{V_{\star}}\right)^{0.89}\left(\frac{20000 \mathrm{AU}}{d_{\odot}}\right)^{1.78},
$$

otherwise, and where $d_{\odot}$ is the impact parameter with respect to the Sun, and $M_{\odot}$ the solar mass.

The full strength of a stellar sequence may be evaluated by estimating the total number of directly injected comets, considering only the case where $N_{\star}>10$ since the two power laws are not very reliable for small values of $N_{\star}$. One can also count the number of stars in a sequence with $N_{\star}>10,100$ or 500 . These characteristics are given in Table 1.

Figure 1 shows for each stellar sequence the value of $N_{\star}$ ( when larger than 100 in order to show only the most effective stellar encounters) versus the time of stellar passage. The stellar type and the impact distance with the Sun are also shown for each star.

Table 1 and Fig. 1 illustrate the wide range of behaviours found using only ten different sequences. The estimated number of comets directly injected during shower periods varies from about 3500 to more than 13000. On Fig. 1 one notes that indeed sequences \#4 and \#7 include several star passages at less than or about $1000 \mathrm{AU}$, whereas sequences \#1, \#5 and \#8 contain just a few moderately effective star passages, all at more than $3000 \mathrm{AU}$.

Regarding the frequency of each stellar type among the rogue stars, for the greatest comet showers one finds from Fig. 1 the following: 2 B0, 3 A0, 5 A5, 11 F0, 5 F5, 6 G0, 8 G5, 9 K0, 8 K5, 3 M0, 1 M5, 6 gi, 


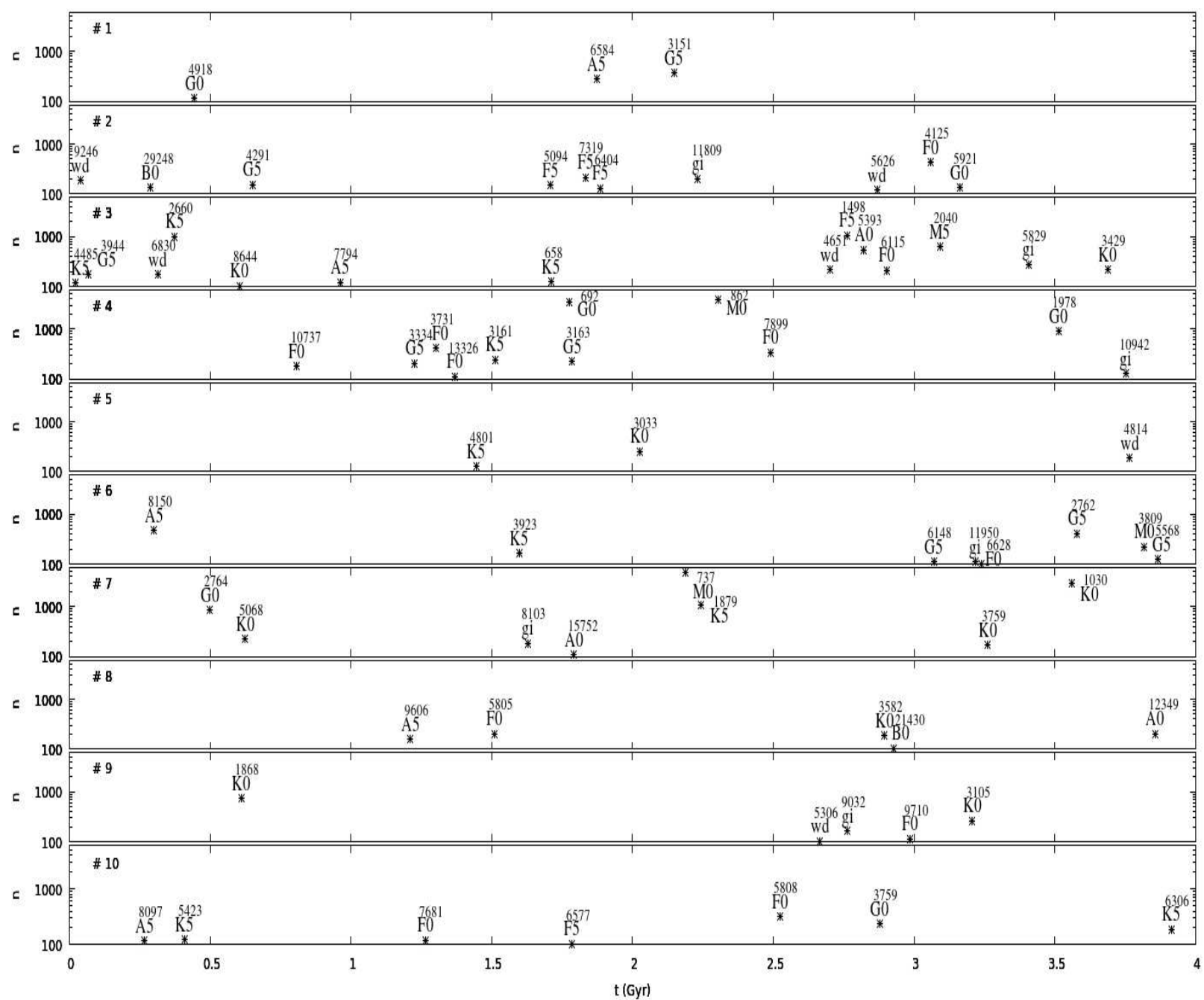

Figure 1: Value of $N_{\star}$ (when larger than 100) versus the time of stellar passage for each stellar sequence (the number at the upper left corner of each plot). The stellar type and the impact distance with the Sun are shown in each case close to the star symbol.

$6 \mathrm{wd}$, for a total of 74 stars. Comparing these numbers to the frequency of stellar passage at less than 1 pc during 1 Myr given in Table 1 of Rickman et al. (2008), the higher efficiency of massive stars to produce comet showers is clearly confirmed.

We have not been able to establish a clear relationship between the characteristics of a stellar sequence as listed in Tab. 1 and the results obtained in the present study. Indeed, because of the strong anisotropy of our initial conditions set, not only the strength but also the geometry of each encounter affect the results significantly. Consequently, from now on we will focus on the results obtained with stellar seq. \#2 that we have considered as a good median case. When needed, the range of the results obtained over our ten stellar 


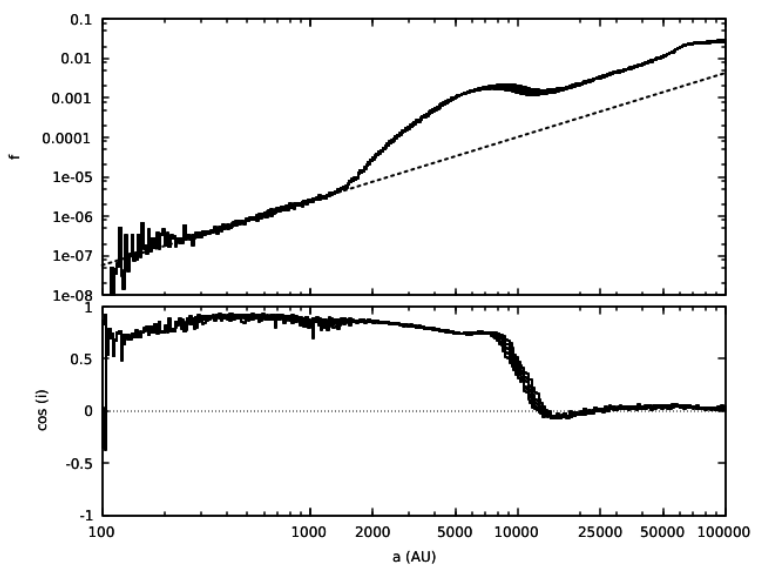

Figure 2: Top panel: distributions of $z=-1 / a$ for the surviving comets at the five different storage times. The dotted line is a linear fit in $\log$ scale to the distributions for $a \in[300,1000 \mathrm{AU}]$ (see text for detail). Bottom panel: behaviour of the median of $\cos i$, where $i$ is the ecliptical inclination, in each bin, versus $a$ for the five different storage times. For both panels, the results for all storage times are overlapping, and the curves are indistinguishable.

sequences will be given.

\section{Final shape of the Oort cloud}

\subsection{With the TP-model}

We will first discuss the final shape of the Oort cloud for the five final snapshots using the TP-model. As mentioned above, the snapshots correspond to five different storage times ranging from $t \approx 4.0 \mathrm{Gyr}\left(17 T_{G}\right)$ to $t \approx 4.9 \mathrm{Gyr}\left(21 T_{G}\right)$. Over this period, the number of comets remaining in the cloud decreases from $2.50 \cdot 10^{6}$ to $2.16 \cdot 10^{6}$ comets. During the whole integration time-span, most of the comets were ejected on hyperbolic orbits. The other end states are as follows: about 650000 reached a semi-major axis smaller than $100 \mathrm{AU}$, for which the evolution stops, about 1900 impacted the Sun and 0, 1, 41 and 100 impacted Jupiter, Saturn, Uranus and Neptune, respectively.

The upper plot of Fig. 2 shows the distributions of $z=-1 / a$ proportional to the orbital energy, where $a$ is the semi-major axis of the surviving comets, at the five different storage times. For clarity of the plot, the distributions are obtained using a fixed bin size in $\log a$, where $a$ is the semi-major axis of the comets expressed in AU. However, because for Oort cloud comets it is convenient to consider $z$ rather than $a$, the number of comets in each bin has been divided by $10^{4}\left(z_{\max }-z_{\min }\right)$ where $z_{\min }$ and $z_{\max }$ are the values of $z$ at the borders of the bin. Then each distribution is normalized so that the sum over all the bins equals one. On the lower plot of Fig. 2, for each distribution, we show the behaviour of the median of $\cos i$, in each bin, versus $a$.

We first note that the distributions of orbital energy and the behaviours of the median of $\cos (i)$ are very similar for the five different storage times. Considering that about 1 Gyr separates the first and the last 
final time, we may conclude that the Oort cloud has reached a kind of steady state. This fact will be used in Sec. 4.

Let us recall that when the comets are injected into the Oort cloud at the beginning of the evolution, the distribution of $z$ is flat and the semi-major axes span the range from 1100 to $50000 \mathrm{AU}$. We then observe on Fig. 2 that a transport of semi-major axis has occurred toward very low values. In addition, as mentioned, the semi-major axes of about 650000 comets have passed the threshold at $100 \mathrm{AU}$.

Because the Galactic tides are unable to change the semi-major axis of a comet, except if it is very large, it is clear that this transport is caused by planetary perturbations. This transport induces a distribution of orbital energy proportional to $|z|^{\beta}$ with $\beta=-1.62 \pm 0.03$ for semi-major axis between 300 and 1000 AU, considering the storage time $t_{s}=19 T_{G}$ (otherwise $\beta$ ranges between -1.70 and -1.62 , with no general trends, confirming the steady state). Such values for $\beta$ are consistent with the value found by Duncan et al. (1987). Below $300 \mathrm{AU}$ the fit is not so good, and it seems that the fitted power law underestimates the number of comets. While outside the scope of this paper, we note that from a further investigation by Saillenfest et al. (2016), it appears that this accumulation may come from comets trapped in mean motion resonances with Neptune associated to a Lidov-Kozai mechanism (see also Gomes et al., 2005).

For semi-major axes larger than $1000 \mathrm{AU}$, the fit remains good until $1500 \mathrm{AU}$, from where the departure from this power law (and, in fact, any power law) is evident. Thus, beyond this threshold the dynamics is not driven by the planets only but also by the Galactic tides.

Indeed, it is well known that the Galactic tides mainly affect the perihelion distance and the Galactic inclination of the comets and that its strength increases with the semi-major axis. Consequently, the larger is the semi-major axis, the higher will be the efficiency of the tides to drive the perihelion of the comets away from the planetary region, thereby protecting the comets from ejection. In other words, the region where the tides are not efficient enough to remove the perihelion from the planetary region gets depleted because of planetary ejection. This roughly explains why the distribution of surviving comets increases with the semi-major axis for $a>1500 \mathrm{AU}$ (at least until about $8000 \mathrm{AU}$ ), whereas initially the distribution is flat in this range.

Then why does the distribution decrease again beyond 8000 AU? Is the knee observed at 1500 AU an artefact of our cut off at 1100 AU for the initial semi-major axis of the comets? To answer these questions we need to consider the population of the surviving comets in the $(a, q)$ plane.

Before this, let us make two points about the behaviour of the median of $\cos (i)$ observed in the lower panel of Fig. 2. Clearly, in the planetary transport regime, between 300 and 1000 AU the inclination remains small since the planets are driving the dynamics. For semi-major axes larger than 1500 AU, the increase of 
the strength of the tides with the semi-major axis is also evident. Below $300 \mathrm{AU}$ the observed increase of the inclination is associated to the trapping of comets in mean motion resonances with Neptune (Saillenfest et al., 2016; Gomes et al., 2005).

It is well known from Heisler and Tremaine (1986) that for moderate semi-major axis, i.e., smaller than about $40000-50000 \mathrm{AU}$, the effect of the tides is well modelled by an integrable system, where the radial component of the tidal acceleration is neglected and the equations of motion are averaged over one orbital period of the comet. In this case, the period of the cycle of the perihelion distance under the tidal action is explicitly obtained from the Galactic orbital elements of the comet (see Appendix B). Let us call $P_{q}$ this period. The number of perihelion cycles that a comet may perform during a time span $t$ is then simply $n_{P}(t)=t / P_{q}$.

In addition, considering the integrable system, it may be shown (see Appendix A) that for orbits with small semi-major axis and high eccentricity, the maximal value $q_{\max }$ that the perihelion distance can reach, starting from $q_{0}$ with arbitrary inclination and argument of perihelion during a time span $t$, is estimated by (as far as $t$ remains small with respect to $P_{q}$ ):

$$
q_{\max }=\left(\sqrt{q_{0}}+\frac{5 \sqrt{2}}{8} \frac{\mathcal{G}_{3}}{\mu^{\prime}} t a^{2}\right)^{2},
$$

where $\mathcal{G}_{3}=4 \pi \mu \rho_{\odot}$ and $\mu^{\prime}=\mu M$ with $\mu$ the universal gravitational constant, $\rho_{\odot}$ the density of the Galactic disk in the solar neighbourhood and $M$ the mass of the Sun plus the giant planets. This is just a global maximum, independent of the way the perihelion distance evolves and whether it is attained by the actual evolution as given by the initial conditions.

Figure 2 shows that the distributions of surviving comets at the five different storage times are very similar. Consequently, we will focus on the intermediate storage time $t_{s}=19 T_{G} \approx 4.5$ Gyr. Fig. 3 shows the repartition of the surviving comets at this storage time, in the $(a, q)$ and $(a, \cos i)$ plane on the middle and bottom panels, respectively. In the top panel, the black line shows the variation of the median of $n_{P}\left(t_{s}\right)$ for $t_{s}=19 T_{G}$, as computed in each semi-major axis bin. The grey area is defined by the lower and upper quartiles of $n_{P}\left(t_{s}\right)$ for each semi-major axis bin. Like in Fig. 2, a uniform bin size has been chosen in $\log a$. In addition, an equal bin size has been chosen in $\log q$ (with $q$ expressed in $\mathrm{AU}$ ) for the $(a, q)$ plane. Then, in each cell, the number of comets has been divided by $10^{4}\left(q_{\max }-q_{\min }\right)\left(z_{\max }-z_{\min }\right)$ for the $(a, q)$ plane and by $10^{4}\left(z_{\max }-z_{\min }\right)$ for the $(a, \cos i)$ plane. Thus, the colour diagrams refer to the number of comets per $\mathrm{AU}$ in perihelion distance and per $10^{-4} \mathrm{AU}^{-1}$ in orbital energy in the $(a, q)$ plane, and to the number of comets per degree for the inclination and per $10^{-4} \mathrm{AU}^{-1}$ in orbital energy in the $(a, \cos i)$ plane. On both colour diagrams, the rectangular area limited by the grey lines shows the location of the initial conditions. 


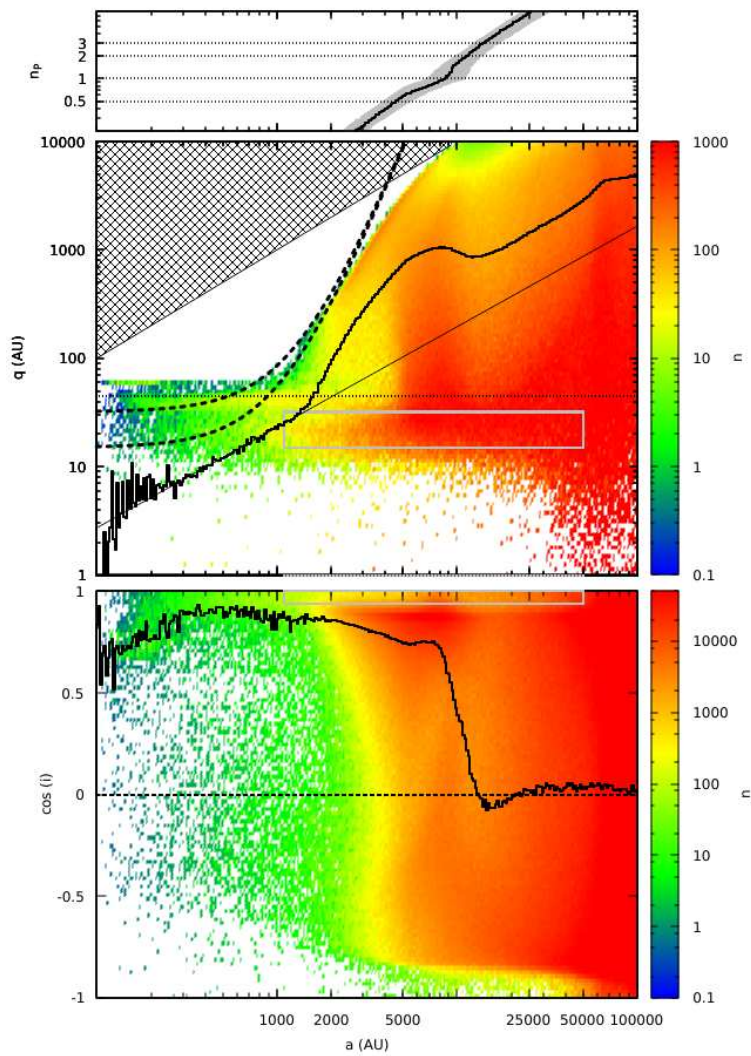

Figure 3: Top panel: variation of the median of $n_{P}\left(t_{s}\right)$ with the semi-major axis $a$ for $t_{s}=19 T_{G}$. The grey area is defined by the 25 th and the 75 th percentiles of $n_{P}\left(t_{s}\right)$. Middle panel: distribution of the surviving comets for $t_{s}=19 T_{G}$ in the $(a, q)$ plane. The full black line and the dotted straight line are the same as the lines shown on Fig. 2 (for $t_{s}=19 T_{G}$ ). For clarity, no vertical axis has been plotted for these lines. The dashed lines give maximal values of the perihelion distance under tidal action only as given by Eq. 3. Bottom panel: distribution of the surviving comets for $t_{s}=19 T_{G}$ in the $(a, \cos i)$ plane. The black line is the same as the one shown on Fig. 2 (for $t_{s}=19 T_{G}$ ). On both colour diagrams, the grey rectangles give the position of the initial conditions. The colour scale is defined to the right of each diagram. 
In addition, on the middle plot, the distribution of comets in orbital energy (black line) and the best fit power law for semi-major axis between 300 and $1000 \mathrm{AU}$ (black thin line) already plotted in Fig. 2 for $t_{s}=19 T_{G}$ have been superimposed on the colour diagram (for clarity, the ordinate axis has not been plotted for this distribution). The maximum possible value that the perihelion distance can reach under the action of the Galactic tides, given by Eq. 3 for $t_{s}=19 T_{G}$, has also been plotted for the two extreme initial values of the perihelion distance $q_{0}=15 \mathrm{AU}$ and $q_{0}=32 \mathrm{AU}$ (dotted lines).

On the bottom panel, the variation of the median of $\cos i$, already shown on Fig. 2, has also been plotted for $t_{s}=19 T_{G}$.

Let us first discuss what is observed in the $(a, q)$ colour diagram. Two regimes are clearly highlighted here: a transport regime governed by planetary perturbations for semi-major axis smaller than $1500 \mathrm{AU}$ and a regime governed by the Galactic tides for semi-major axis larger than this.

The maximum value reached by the perihelion distance, given by Eq. 3, under the tidal action fits the data very well for semi-major axes between 800 and 2500 AU. This explains the transition between the two regimes: when the perihelion is out of reach for planetary perturbations, the transport caused by planetary perturbations stops and only the tides will shape the distributions of orbital elements of such comets. Since Eq. 3 is valid only for highly eccentric orbits and for $t_{s}$ small with respect to the period $P_{q}$ of the perihelion cycle, the fit gets worse for increasing semi-major axis, because $P_{q} \propto P^{-1}$, where $P$ is the orbital period of the comet.

The fact that the fit is very good between 800 and $2500 \mathrm{AU}$ shows that the location of the transition is not an artefact of our limiting initial semi-major axis at $1100 \mathrm{AU}$. The knee observed around $a=1500 \mathrm{AU}$ in the orbital energy distribution is a consequence of the shift between the planetary transport regime and the tidal regime. An inner edge to the innermost part of the Oort cloud was already observed in Duncan et al. (1987) and Dones et al. (2004), however at a slightly higher semi-major axis ( $a \sim 2-3000 \mathrm{AU})$.

In the present study, the location of the knee is well understood considering that a transport of semimajor axis caused by the planets can take place only if the perihelion distance is smaller than 45 AU. Equation 3 tells that for our initial conditions and for $t_{s}=19 T_{G}$, a semi-major axis greater than $543 \mathrm{AU}$ if $q_{0}=15 \mathrm{AU}$ or $891 \mathrm{AU}$ if $q_{0}=32 \mathrm{AU}$ is required for the tides to remove the comets from this transport regime. Considering the median value of the perihelion distance $q_{\text {med }}$ instead of the maximum (see Appendix A), these two semi-major axis thresholds become $1090 \mathrm{AU}$ and $1640 \mathrm{AU}$, respectively. These values correspond quite well to the location of the knee.

We now turn to the main features observed in the tidal regime of the $(a, q)$ diagram in Fig. 3. Between 5000 and $10000 \mathrm{AU}$ of semi-major axis, the distribution of orbital energy has a local maximum. These 
comets are on the decreasing branch of their perihelion cycle. When the cycle is complete (judging from the top panel, this occurs mainly for semi-major axis between 7000 and $11000 \mathrm{AU})$, the perihelion is back in the planetary region, where comets are affected again by the planets. Returning to the top panel, we note that, between $10000 \mathrm{AU}$ and $14000 \mathrm{AU}$, many comets had enough time to perform two complete perihelion cycles, bringing them back to the planetary region for the second time. This range of semi-major axis agrees rather well with the decrease observed in the distribution of orbital energy for semi-major axes between 8000 and $13000 \mathrm{AU}$.

Between 13000 and 50000 AU of semi-major axis, comets with similar semi-major axes may have performed different numbers of perihelion cycles, as seen from the top panel of Fig. 3. Hence, the imprint of the tides becomes more confused. However, the increase in the distribution of orbital energy within this range may be explained by the fact that for increasing semi-major axis, the time spent by the perihelion in the planetary region decreases, and this leaves less chance for the planets to affect the orbital evolution of the comets. For semi-major axes larger than $40000 \mathrm{AU}$, the time spent may be zero, i.e. the perihelion gets into and out of the planetary region while the comet itself is far from perihelion, so that the planets cannot perturb the comet during the transit of the perihelion through the planetary region (what we called the "timing problem", see Fouchard et al., 2010).

Concerning comets with semi-major axis larger than $50000 \mathrm{AU}$, since the tides cannot change significantly their semi-major axes, they clearly have been placed there by planetary perturbations.

As regards the tidal regime in the $(a, \cos i)$ plane (bottom diagram in Fig. 3), we notice that a median value of $\cos i$ close to zero is not synonymous with an isotropic Oort cloud. In particular, for semi-major axes between 20000 and $50000 \mathrm{AU}$, the median is close to zero, whereas the cloud is clearly not isotropic since the population of retrograde orbits coplanar with the ecliptic is almost non-existent. Indeed, Higuchi et al. (2007) show that for an initial ecliptical inclination close to zero, it is difficult to reach an inclination larger than $153^{\circ}$ under the tidal effect only.

This anisotropy is expectable, since the tides induce an almost integrable dynamics. The only exception is for semi-major axes larger than 50000 AU, where the cloud seems to be isotropic. For such large semimajor axis, the tides cannot be considered as a quasi integrable system since the two hypothesis upon which the integrability rely are broken. Indeed, the radial component may not be negligible and the mean anomaly cannot be considered as a fast angle any more. In addition, considering that all the comets in this region have been placed there by the planets, this gives an additional contribution to the failure of the integrability. The loss of integrability contributes to the apparent isotropy of the cloud beyond $50000 \mathrm{AU}$.

It is out of the scope of the present study to show a realistic picture of the planetary transport regime. 
Our model does not include planetary perturbations for perihelion distances larger than $60 \mathrm{AU}$. This is clearly the reason for the absence of comets beyond this value for semi-major axes smaller than 1000 AU. We are still able to draw some conclusions:

- the threshold at $45 \mathrm{AU}$ (see Brasser and Schwamb, 2015, for instance) for the planetary perturbations to induce a transport in semi-major axis is clearly observed;

- the accumulation of comets with perihelion close to $60 \mathrm{AU}$ for semi-major axes smaller than $300 \mathrm{AU}$ is caused by the interaction between mean motion resonances with Neptune and a Lidov-Kozai cycle induced by the planets (Gallardo et al., 2012; Gomes et al., 2005). This is investigated in Saillenfest et al. (2016).

We claim that the limit at $60 \mathrm{AU}$ for the planetary perturbations affects neither the shape of the cloud at more than 1500 AU from the Sun nor the production of observable "new" comets (which will be discussed in Sec 4). Indeed, it has been observed that for semi-major axis greater than $1500 \mathrm{AU}$, the tides dominate the evolution of the perihelion distance and for smaller semi-major axis it is quite unlikely that a comet with perihelion beyond $60 \mathrm{AU}$ from the Sun becomes observable, i.e., that its perihelion distance decreases to less than $5 \mathrm{AU}$.

\subsection{With the full models}

Now we consider the case where stellar perturbations are also at work. Ten simulations have been performed using different sequences of stellar encounters. As mentioned in Sec. 2, we will not discuss the effects of each stellar sequence.

Model \#2 will be used as a canonical case of a full model, whereas the set of ten simulations is used to estimate the range of validity of our results. Sequence \#2 is a rather "quiet" sequence, in the sense that no individual stellar passage seems to have strongly influenced the final shape of the Oort cloud.

Over the five storage times, the number of comets remaining in the cloud decreases from $3.04 \cdot 10^{6}$ to $2.72 \cdot 10^{6}$. As for the TP-model, most of the lost initial comets were ejected on hyperbolic orbits. About 600000 reached a semi-major axis smaller than $100 \mathrm{AU}$, about 1400 (1260 - 1639 considering all sequences) impacted the Sun and 1 (0-2), 1 (0-3), 39 (32-45) and 77 (73-105) impacted Jupiter, Saturn, Uranus and Neptune, respectively.

In comparison to the TP-model, we note that including the stellar perturbations protects the comets from ejection and collision with the Sun. At first glance, this may seem contradictory, since the stars may inject comets on their own into the planetary region. However, our initial comets have all their perihelia in the planetary region, and the effects of Galactic tides being almost integrable, the perihelia of the comets 
will tend to return to this region, until an ejection or a collision occurs. When stellar perturbations are at work, the perihelion cycle caused by the tides may be broken so that the perihelia are lifted away from the planetary region, thus protecting the comets from the considered end states.

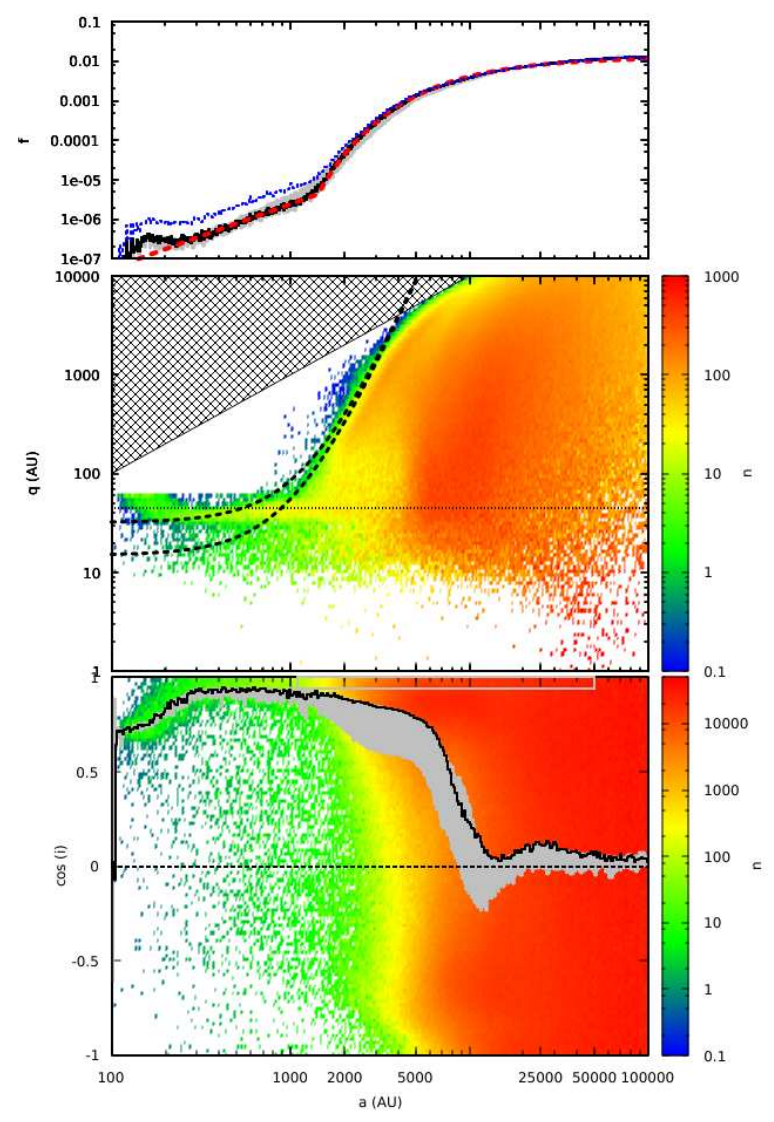

Figure 4: The top panel shows the distribution of orbital energy in Model \#2 at the five storage times (these distributions are overlapping). The grey area (also almost indistinguishable) corresponds to the range obtained using the ten full models for $t_{s}=19 T_{G}$. The blue line shows the distribution obtained at $t_{s}=19 T_{G}$ using only the sample of Model \#2 comets that were decoupled at some time during their evolution. The dashed red line shows a fit to the distributions using two analytic laws (see text for details). The two colour diagrams are the same as in Fig. 3. In the ( $a$, cos $i)$ plane, the grey area shows the range of the median of $\cos i$ obtained with the ten full models.

The top panel of Fig. 4 shows the distribution of orbital energy for the surviving comets at the five storage times. These distributions were obtained in the same way as for Fig. 2. All five distributions overlap and are barely distinguishable. This shows that, as for the TP model, the cloud has reached a sort of steady state.

The grey area in the top panel of Fig. 4 indicates the range of all the distributions obtained with the ten different stellar sequences at the storage time $t=19 T_{G}$. We see that the global behaviour of the energy distribution is quite robust with respect to the individual history of stellar encounters, in particular with the transition between the planetary transport and Galactic tide regimes always located between 1000 and 
$2000 \mathrm{AU}$.

Like for the TP model, the orbital energy distribution may be fitted by a power law for semi-major axis between 300 and $1000 \mathrm{AU}$. For Model \#2 and $t=19 T_{G}$, the distribution is proportional to $|z|^{\beta}$ with $\beta=-1.63 \pm 0.04$. Considering the four other storage times for this stellar sequence, the index $\beta$ ranges between -1.63 and -1.70 , and considering all ten models for $t_{s}=19 T_{G}, \beta$ ranges between -2.18 and -1.57 . We note that $\beta$ is quite dependent on the stellar sequence. Models $\# 3$, \#4 and \#7 yield $\beta$ values close to -2 . According to Tab. 1 and Fig. 1, these sequences are rather "hot" in the sense that they contain events that efficiently inject comets directly into observable orbits. However, Model \#8, which represents a "quiet" case, also yields a value of $\beta$ close to -2 . This highlights the difficulty to relate the effects of stellar sequences to their measurable characteristics.

For semi-major axis greater than 2000 AU, we are out of the planetary transport regime, and the distribution of orbital energy is shaped mainly by the effect of the Galactic tides. Compared to the TP model, we note that the inclusion of stellar perturbations smoothens the energy distribution. In particular, the local minimum observed on Fig. 2 at $a=13000 \mathrm{AU}$ is not present any more. The distribution can now be fitted by a Boltzmann law, i.e., a function proportional to $10^{\alpha z}$. It was not possible to fit the orbital energy distribution by a single power law for semi-major axis greater than 2000 AU, whereas this was possible considering an exponential formula ${ }^{2}$. For Model $\# 2$ and $t_{s}=19 T_{G}$, we get $\alpha=4952 \pm 13$ for surviving comets with semi-major axis between 2000 and 50000 AU. Considering the four other storage times, $\alpha$ decreases from 5074 to 4864 . The decrease is small but indicates that the distribution flattens with time. It indicates that the steady state is slowly evolving.

Let us consider only the comets for which the semi-major axis has reached a value less than 1000 AU at some time during their evolution. In a former paper (Fouchard et al., 2014b) these comets were called "decoupled" comets, because their semi-major axis was too small for the tides or the stars to significantly affect their dynamics during the age of the solar system. The orbital energy distribution obtained from these comets corresponds to the blue dotted line on the top panel of Fig. 4. Surprisingly, for $a>2000$ AU, the distribution agrees very well with that obtained considering all the comets. If we perform a fit only from these comets, we get $\alpha=4628$, i.e., the distribution is slightly flatter than that of all the comets. It appears that this group of comets is very active because, and this is crucial, their perihelia are close to or inside the planetary region. Consequently, they may still suffer large variations of their semi-major axis because of the

\footnotetext{
${ }^{2}$ This is in fact the Boltzmann distribution, known from thermodynamics and statistical mechanics to characterize a fully relaxed dynamical system. In Jeans (1919), the distribution of orbital energy for such system corresponds to a exponential law times a power law. In the present study, a simple exponential law was used and produced very good fit to the distributions obtained
} 
planetary perturbations, putting them back under the influence of tides and stars.

Boltzmann distributions have been fitted for $a \in[2000,50000] \mathrm{AU}$ and for $t_{s}=19 T_{G}$ considering all the ten models. We find that $\alpha$ ranges between 5653 and 4798 . We note that the effect of the stellar sequences is now much smaller than it was in the planetary transport regime, i.e., for $a$ between 300 and $1000 \mathrm{AU}$.

In the top panel of Fig. 4 , the dotted red line shows the Model \#2 distribution for $t_{s}=19 T_{G}$, as fitted by the power law and the exponential law. This fit is indeed excellent in both regimes, and moreover, this is the case for all stellar sequences.

The middle and bottom panels of Fig. 4 show the repartition of surviving comets for Model \#2 at $t_{s}=19 T_{G}$ in the $(a, q)$ and $(a, \cos i)$ planes, respectively. In the $(a, q)$ plane, the maximum value that the perihelion distance can reach at this time, starting from initial values of 15 and $32 \mathrm{AU}$ under the action of an integrable tide only as given by Eq. 3, are also plotted versus the semi-major axis. In the $(a, \cos i)$ plane, the black line shows the behaviour of the median of $\cos i$ versus semi-major axis, and the grey area shows the range covered by the ten medians obtained from the different stellar sequences.

The tidal regime observed on the $(a, q)$ diagram of Fig. 4 differs from the one observed on Fig. 3, where the stars were not at work, in two aspects.

- Because of the stellar contribution, some comets have perihelion distances larger that the maximum allowed under the tidal action only.

- In the model without stars (Fig. 3) many comets were back into the planetary region after one perihelion cycle, and this occurred mainly for semi-major axis between 7000 and 11000 AU. However, these comets are not back into the planetary region in the full model. The stellar perturbations may break the tidal perihelion cycle, and thus, the minimum of the perihelion after one cycle may be out of this region. This explains why we no longer see the local minimum around $13000 \mathrm{AU}$, which was observed in Fig. 3.

The behaviour of the median of $\cos i$ versus $a$ shown at the bottom of Fig. 4 tells us that the Oort cloud is certainly not isotropic for semi-major axes below $8800 \mathrm{AU}$, whatever is the stellar sequence used. This value corresponds to the minimum semi-major axis, for which at least one median is equal to zero. In addition, one notes that the spread of the median obtained over the ten stellar sequences is rather larger for semi-major axis smaller than $10000 \mathrm{AU}$. This is consistent with the observation made by Higuchi and Kokubo (2015) that stellar perturbations are much more efficient to affect the inclination rather than the orbital energy. 
Before considering the production of observable "new" comets, we first have to discuss the population of the so called Tidally Active Zone (TAZ). This region is the phase space domain of the Oort cloud from where the nearly integrable Galactic tide is able to make a comet observable on its own, i.e., the minimum of the perihelion distance over one cycle is smaller than 5 AU (Fouchard et al., 2011a). The density of comets inside this region is crucial in order to explain the flux of observable comets. Consequently, it is important to study the filling of the TAZ in order to evaluate and understand this flux. By filling we mean the fraction of comets belonging to the TAZ within any semi-major axis bin.

Figure 5 shows the filling of the TAZ versus the comet semi-major axis for the TP model. We considered the sample of surviving comets at storage time $t_{s}=19 T_{G}$. In addition, we plot the TAZ fillings of our initial condition sample and a thermalized sample of initial conditions, i.e., isotropic and with a distribution of eccentricity $e$ proportional to $e$.

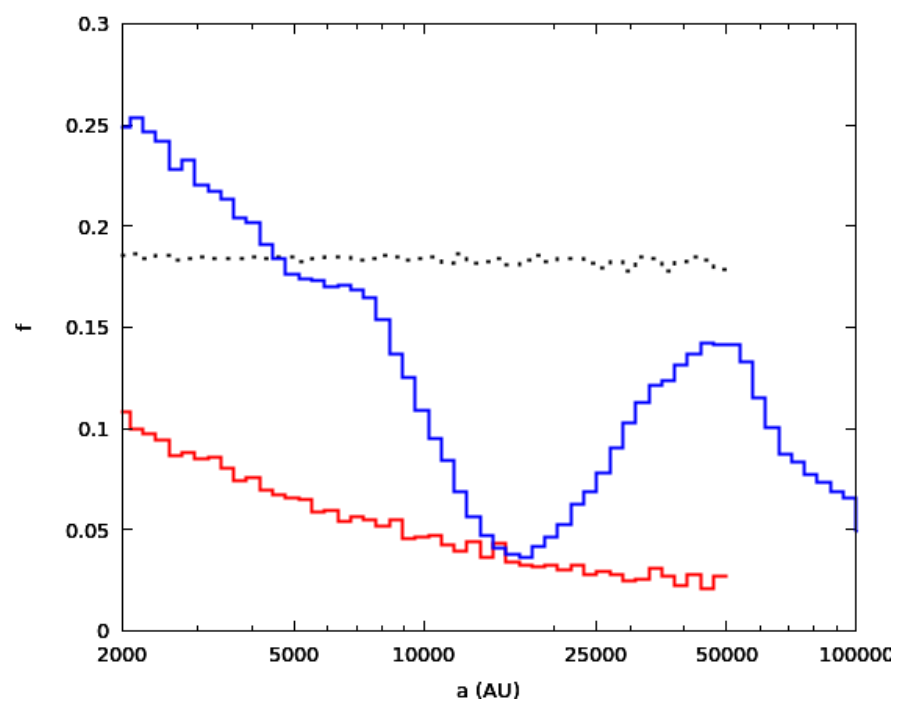

Figure 5: Fraction of comets in the TAZ versus the semi-major axis for the TP-model (blue line), our initial conditions (grey dotted line) and a thermalized set of comets (red line).

For our initial conditions, the TAZ filling is almost independent of the semi-major axis (about 18\%) and higher than the maximum value obtained with the thermalized cloud in the same semi-major axis range. These two properties are a consequence of our choice for the initial conditions. The initial perihelion distance distribution is uniform between 15 and $32 \mathrm{AU}$, i.e. close to the threshold chosen to define the TAZ (remember, however, that the TAZ is defined by the minimal value of the perihelion distance over a full cycle generated by an integrable galactic tide); this explains the higher TAZ filling obtained with our set of initial conditions with respect to the thermalized cloud. 
Moreover, the eccentricity does not vary strongly for our initial conditions (the median of the eccentricity from $a=1100 \mathrm{AU}$ to $a=50000 \mathrm{AU}$ increases from 0.9786 to 0.9995 , i.e., about $2 \%$ ), whereas the distribution properties of the other orbital elements that influence the maximum eccentricity reached over one cycle induced by the Galactic tides (see Appendix B) are constant throughout the cloud. Consequently, the maximum eccentricity varies little with the semi-major axis. Since it determines whether an orbit belongs to the TAZ or not, we see why the TAZ filling is almost constant throughout our initial population.

The TAZ depletion for the TP-model is easily explained as follows. The probability of ejection from the Oort cloud by the giant planets is a decreasing function of the perihelion distance. This probability is close to $90 \%$ for comets entering the observable region, i.e., at less than 5 AU from the Sun and about $10 \%$ for perihelion close to 15 AU (Fouchard et al., 2013). Since comets inside the TAZ have a minimal perihelion distance over one cycle smaller than 5 AU, they are more affected by planetary ejection than comets outside the TAZ. Obviously, the ejection is also more likely when the perihelion is close to its minimal value over one cycle.

In the top panel of Fig. 3, it is seen that for semi-major axis between 7000 and 11000 AU, in most cases the perihelion had time to compute a complete cycle, i.e., to reach its minimal value, and that for higher semi-major axis the perihelion had time to complete several cycles. Consequently the higher is the semi-major axis, the higher is the probability for a comet inside the TAZ to be ejected from the Oort cloud by planetary perturbations. This relation holds until $a \sim 20000 \mathrm{AU}$.

For $a>20000 \mathrm{AU}$, the TAZ filling increases again. This is mainly caused by the perihelion-passagetiming problem (Fouchard et al., 2010), which means that the perihelion distance can drift through the observability region while the comet is far from perihelion. In such a case the comet is protected from planetary perturbations when the perihelion goes through its minimal value. Since the perihelion drifts faster for increasing semi-major axis, this timing problem gets stronger for higher semi-major axis.

One may wonder, however, why for semi-major axes from 2000 to $5000 \mathrm{AU}$, the TAZ is even more filled than at the beginning? This is because the perihelion cycle is typically longer for the comets inside the TAZ than for those outside the TAZ for our set of initial conditions (see Appendix B for details). Hence, if the period is longer, the comet runs less of a risk to enter into the planetary region, and thus of being ejected by planetary perturbations.

It is remarkable that when $a>20000 \mathrm{AU}$, the TAZ filling for the TP-models is larger than the one obtained for a thermalized Oort cloud. This is an indication that, at least when stars are not at work, the Oort cloud is not isotropic even at such distances from the Sun. Indeed, although it was observed in Fig 3 that the median value of the cosine of the ecliptic inclination is close to zero when $a>25000$ AU for the 


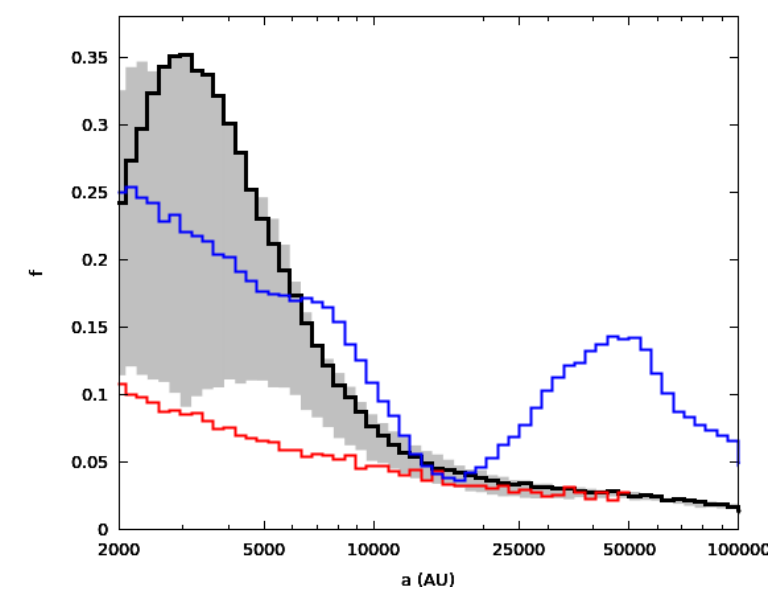
formed. An isotropic cloud would not have such an affinity. at $t_{s}=19 T_{G}$.

Figure 6: TAZ filling versus semi-major axis for Model \#2 (black line), the TP-model (blue line) and the thermalized sample of comets (red line). The grey area shows the range of filling obtained using all the ten models. All data (except for the red line) are obtained at $t_{s}=19 T_{G}$.

TP-model, this was mainly caused by the integrable part of the Galactic tide involving a Galactic inclination cycle (related to the perihelion cycle) and a precession of the Galactic longitude of the ascending node of the cometary orbit (Breiter et al., 1996; Levison et al., 2006). This process does not affect the adherence of a comet to the TAZ, and consequently, the cloud preserves the affinity for the TAZ with which it was

For comets with $a>50000 \mathrm{AU}$, the TAZ filling decreases again, since at such large distances the approximation of the tidal dynamics by an integrable system fails completely.

Let us now turn to the behaviour of the TAZ filling when stellar perturbations are at work, as shown in Fig. 6. The black line corresponds to our canonical case using Model \#2, and the grey area indicates the range of fillings obtained over all the ten models. Again, the fillings are obtained for the surviving comets

Two regions are clearly identified.

- For semi-major axes between 2000 and $5000 \mathrm{AU}$, the TAZ filling is very sensitive to the stellar sequences. We know that for this range of semi-major axis, the comets did not have time to perform a complete perihelion cycle, while the perihelia have been removed from the region where planetary perturbations are efficient. Consequently, this is the region where stellar perturbations are the only factor able to change the TAZ affinity. The large differences are clearly an indication that this effect is caused by only few stars, which differ strongly from one sequence to another. Because the geometry of the encounter is also important, it is not possible to draw any further conclusion. Indeed, Model \#7 yields a TAZ filling very close to the isotropic case, whereas Model \#4 yields one of the highest fillings. 
However, looking at Tab. 1 and Fig. 1, both these sequences are seen to contain some of the most efficient shower making stars.

- For semi-major axes larger than 7000 AU, all TAZ fillings converge toward the isotropic case, showing now that background stars are more important than individual stars. Between 7000 and about $13000 \mathrm{AU}$ the decrease of the TAZ filling is also caused by the planets, since comets in the TAZ may have their perihelia back into the planetary region giving a chance to eject them from the Oort cloud. Beyond 13000 AU, the TAZ filling does not increase again like in the TP-model, since the stars have now fulfilled their role by cancelling the TAZ affinity of the comets, also making the cloud nearly isotropic (see Fig. 4).

\section{The observable comets}

\subsection{The flux of "new" comets}

As we have seen, the distributions of comets in the Oort cloud at the five different storage times share the same statistical characteristics. However, due to the effects of passing stars and planets - each of which can be considered as a stochastic process - during the time elapsed between consecutive storage times $\left(1 T_{G} \approx 236 \mathrm{Myr}\right)$, we may consider that the five storage sets are mutually independent.

Consequently, the observable comets coming from each storage set may be considered as coming from different initial population. Hence, the whole set of observable comets corresponds to a final flux of observable comets using $5 \times 10^{7}$ initial conditions. The observable time is then taken as the median of all storage times, i.e., $t=19 T_{G} \approx 4.49$ Gyr.

We recall that during its evolution a comet may be ejected from the cloud into interstellar space, impact the Sun or a planet or have a semi-major axis smaller that $100 \mathrm{AU}$, under which conditions the evolution stops. If it survives all these end states, it may become an observable comet.

For this to occur, a comet that has been stored must pass at less than 5 AU from the Sun at its first perihelion passage after an additional relaxing period lasting $\Delta_{R}=30 \mathrm{Myr}$. The role of the relaxing period is to remove any shower making stars, so that our set of observable comets may be considered as quiescent according to the terminology used in Fouchard et al. (2011b).

The observable comets do not have equal importance. We have to weigh each of them by the probability that the perihelion passage occurs during a fixed observing period. If this period is set equal to $1 \mathrm{Myr}$, then each observable comet is replaced by a number of comets per million years, so that the weight to be applied is $10^{6} / P_{\text {orig, }}$, where $P_{\text {orig }}$ is the orbital period of the observable comet (counted in years), as it enters the planetary region during its observable perihelion passage (this is usually called the "original" orbital period). 
We now pay attention to the flux of "new" comets, i.e., observable comets with an original semi-major axis larger than $10000 \mathrm{AU}$. We will focus on the set of new comets for mainly two reasons.

- The set of observable comets with original semi-major axis less than $10000 \mathrm{AU}$ is statistically unstable. Because of their large weights, few observable comets with small semi-major axis would have a drastic effect on the results.

- Preliminary tests of how fading may affect the distribution of observable comets have shown that a fading law taken from the best fit in Wiegert and Tremaine (1999) does not affect the distribution of the new comets sample, whereas it does affect the distribution of comets with smaller semi-major axis. Therefore, limiting ourselves to the new comets sample allows us to consider our results as independent of fading, at least as the one proposed by Wiegert and Tremaine (1999).

The observed flux has been estimated to be about 4 comets per year for perihelion distance smaller than $5 \mathrm{AU}$ with a total absolute magnitude $H_{T}<11$ (Francis, 2005). Many estimates of the number of objects in the actual Oort cloud population necessary to supply the present flux of observed "new" comets result in about $(1-5) \cdot 10^{11}$ objects (Francis, 2005; Kaib and Quinn, 2009). Considering that in our simulations we have from $70 \%$ to $80 \%^{3}$ of the comets that are ejected during the integration time span, a present population of $(1-5) \cdot 10^{11}$ objects requires an initial population between $3.3 \cdot 10^{11}$ and $2.5 \cdot 10^{12}$. Our results will be calibrated as if the initial population was of $10^{12}$ objects. Thus, to convert our flux of comets per Myr using our sample of 50 million comets to a flux per year considering an initial population of $10^{12}$ objects, we simply have to multiply our flux by 0.02 .

We will also consider the four different classes of observable comets defined in Fouchard et al. (2014a). These classes are:

- the jumpers, consisting of comets for which the perihelion distance passed from values larger than $15 \mathrm{AU}$ into the observable region during the last orbital period before being observable;

- the creepers, consisting of comets whose perihelion distance was less than $15 \mathrm{AU}$ at the perihelion passage taking place one orbital period before the observability;

- the Kaib and Quinn jumpers, consisting of jumpers for which the quantity $z=-1 / a$ (proportional to the orbital energy) increased by more than $10^{-4} A U^{-1}$ at the perihelion passage preceding the observability;

\footnotetext{
${ }^{3}$ This would suggest a very efficient Oort Cloud formation efficiency of $20-30 \%$. However, it refers to our initial disk with an artificial, uniform energy distribution and cannot be used as a realistic estimate.
} 


\begin{tabular}{c|c|cccc|c|c|cc} 
mod. & $\mathrm{c} / \mathrm{yr}$ & $\mathrm{j}$ & $\mathrm{c}$ & $\mathrm{KQ} \mathrm{j}$ & $\mathrm{KQ} \mathrm{c}$ & $a_{0_{50}}$ & ret. & $a_{\text {orig }_{25}}$ & $a_{\text {orig }_{50}}$ \\
\hline 1 & 4.5 & 44.9 & 17.4 & 1.2 & 36.5 & 2412.3 & 53.3 & 21092.6 & 28081.4 \\
2 & 4.5 & 41.4 & 17.8 & 1.3 & 39.6 & 2497.9 & 49.9 & 22097.9 & 28157.0 \\
3 & 3.3 & 41.4 & 17.6 & 1.1 & 39.9 & 2208.5 & 56.5 & 21198.6 & 27828.0 \\
4 & 3.3 & 39.8 & 17.3 & 0.9 & 42.1 & 2230.3 & 55.0 & 21837.1 & 27445.2 \\
5 & 5.1 & 40.2 & 19.2 & 0.4 & 40.2 & 2253.6 & 54.4 & 20623.0 & 27256.7 \\
6 & 3.9 & 44.7 & 16.4 & 1.3 & 37.7 & 2431.9 & 51.8 & 22189.7 & 28562.6 \\
7 & 3.2 & 41.8 & 16.4 & 1.2 & 40.5 & 2343.6 & 57.6 & 20120.4 & 26963.0 \\
8 & 4.0 & 43.6 & 19.1 & 0.8 & 36.4 & 2395.9 & 57.6 & 21501.7 & 28634.6 \\
9 & 4.4 & 42.3 & 18.7 & 0.7 & 38.3 & 2519.9 & 55.7 & 22044.8 & 28458.8 \\
10 & 5.3 & 44.1 & 19.4 & 0.9 & 35.6 & 2328.4 & 55.4 & 21400.6 & 27801.9 \\
\hline $\mathrm{TP}$ & 8.2 & 69.8 & 14.1 & 2.4 & 13.6 & 2266.8 & 50.6 & 29660.7 & 41470.6
\end{tabular}

Table 2: For each sequence of stellar encounters given in the first column, we list the number of observable new comets/yr passing at less than $5 \mathrm{AU}$ from the Sun considering an initial population of $10^{12}$ comets, and for these comets the fractions (in $\%$ ) of jumpers, creepers, KQ jumpers and $\mathrm{KQ}$ creepers, the median value of the initial semi-major axis $a_{050}$, the fraction (in $\%$ ) of retrograde comets, and the first quartile $a_{\mathrm{orig}_{25}}$ and the median $a_{\mathrm{orig}}$ of the original semi-major axis.

- and the Kaib and Quinn creepers, as Kaib and Quinn jumpers but for creepers.

The term Kaib and Quinn comes from Kaib and Quinn (2009), where the process in question was highlighted for the first time in the frame of Oort cloud dynamics.

Table 2 gives, for each stellar sequence and for the TP-model, the number of observable new comets per year passing at less than $5 \mathrm{AU}$ from the Sun considering an initial population of $10^{12}$ comets, and for the set of observable comets the fractions of jumpers, creepers, KQ jumpers and KQ creepers, the median value of the semi-major axis $a_{0_{50}}$, the fraction of retrograde comets, and the first quartile $a_{\text {orig }_{25}}$ and the median $a_{\text {orig }_{50}}$ of the original semi-major axis.

The production rates of comets with all models including stars are consistent with the estimate made by Francis (2005). However, between the lowest production rate $(3.2 \mathrm{com} / \mathrm{yr})$ and the highest one (5.3 $\mathrm{com} / \mathrm{yr}$ ), we have a factor 1.6. Relating these numbers to the global efficiencies of stellar sequences given in Tab. 1, we note that the stellar sequences that contain very efficient shower making stars, i.e., \#3, \#4 and \#7, yield the lowest fluxes (all smaller than 3.3). The other sequences yield fluxes larger than 3.9, but it is hard to relate their production rates to their efficiencies. Thus, it appears that for sequences containing stellar encounters that are disruptive to the Oort cloud, the final flux of observable comets is significantly reduced. The background stellar encounters are also seen to reduce this flux, since the TP-model produces about twice as many observable comets as the average full model.

To explain these differences, we must recall that the flux of observable new comets is closely related to the population of the TAZ (Fouchard et al., 2011a). As seen in Sect. 3.3, for the present simulations the TAZ is initially over-filled with respect to the thermalized case. Hence, stellar perturbations tend to empty 
the TAZ, implying a decrease of the flux of new comets.

Figure 6 showed that for semi-major axis beyond $13000 \mathrm{AU}$, the TAZ in the TP-model is much more filled than with any model including stellar perturbations. Remarkably, due to the different initial conditions, the long term effect of stellar perturbations therefore now works in the opposite sense compared to what we found in former papers (Rickman et al., 2008; Fouchard et al., 2011a).

Regarding the fraction of comets in each class, we note that all models including stellar perturbations give similar results. However, we observe a close correlation between the characteristics of the stellar sequence and the fraction of jumpers and Kaib and Quinn creepers. In particular, although the data are clearly too sparse to propose a general rule, it seems that the fraction of jumpers is smaller for the "hot" Models \#3, \#4 and \#7 than for the other ones, and the opposite holds for the Kaib and Quinn creepers. This trend is evident for the TP-model, where the jumper class clearly dominates. The feature will be explained in Sect. 4.2 .

The fractions obtained for the classes are similar to those observed in Fouchard et al. (2014a). The differences mainly come from different choices of initial conditions. For instance, in Fouchard et al. (2014a) the initial semi-major axis was larger than $3000 \mathrm{AU}$ and the population was fully thermalized. In any case, it appears that between 55 and $60 \%$ of the observable comets had their preceding perihelion at less than 15 AU from the Sun, when stellar perturbations are at work. This is consistent with the study made by Dybczyński and Królikowska (2011). The fraction drops to less than 30\% for the TP-model, and again, this difference will be explained in Sec. 4.2.

The median of the initial semi-major axis of the observable comets is always between 2200 AU and 2520 AU, including the TP-model. This highlights the key role of the planets to diffuse the semi-major axis of the comets. We will return to this point in Sec. 5 .

Concerning the retrograde fraction among the new comets, the percentages shown in the Table indicate a small preference for retrograde orbits (except for our canonical Model \#2). They are consistent with those of Kaib and Quinn (2009), whose initial Oort cloud was rather flattened, and somewhat smaller that what we found in Fouchard et al. (2014a).

\subsection{The Oort spike}

The histograms in the upper panels of Fig. 7 show the distribution of orbital energy of new observable comets, while the fraction of retrograde comets in four ranges of semi-major axis is shown by the black lines of the lower panels. This is done for the TP-model (left panels) and Model \#2 (right panels). In the histograms, each bin is coloured according to the fractions of comets in each class: red for jumpers, blue for 

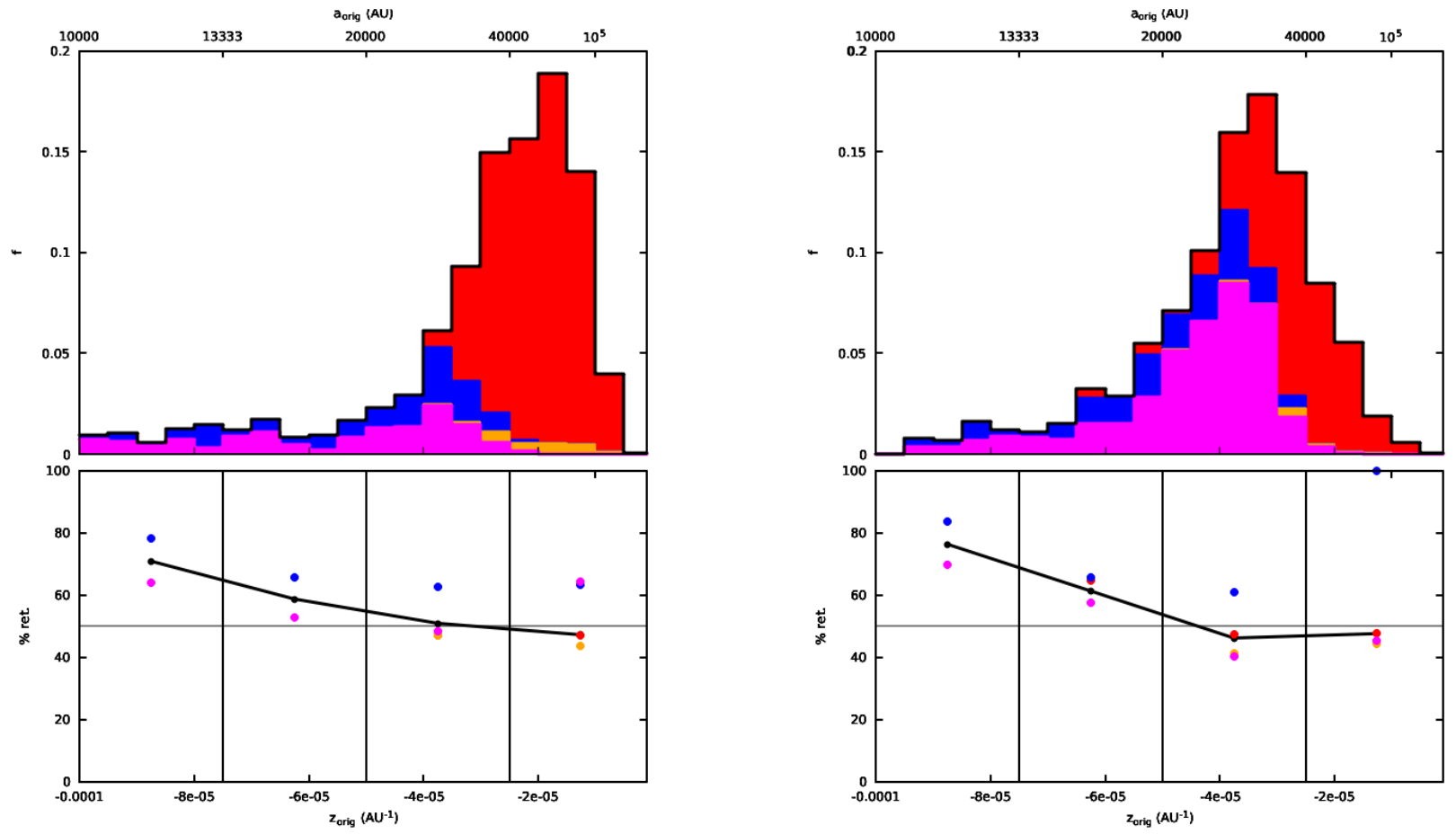

Figure 7: Upper panels: histograms showing the normalized distributions of the original orbital energy $z_{\text {orig }}=-1 / a_{\text {orig }}$ for the TP-model (left) and Model \#2 (right). Each bin is coloured according to the fractions of comets in each class (jumpers in red, creepers in blue, KQ jumpers in orange and KQ creepers in magenta). Lower panels: fractions of retrograde comets, shown by black lines, in four ranges of semi-major axis (given by the vertical lines) for the TP-model (left) and Model \#2 (right). When available, the fraction of retrograde comets in each class is also plotted by a small dot using the same colour code as for the top panels.

creepers, orange for Kaib and Quinn jumpers, and magenta for Kaib and Quinn creepers. The same colour codes are used to plot the fractions of prograde comets in each class in the lower panels, when available.

The Oort spike differs markedly between the two models. The maximum of the spike is at about $57000 \mathrm{AU}$ for the TP-model and about $31000 \mathrm{AU}$ for the model including stellar perturbations. From Tab. 2, we find values for the median $a_{\text {orig }_{50}}$ and the first quartile $a_{\text {orig }_{25}}$ amounting to $a_{\text {orig }_{50}}=41500 \mathrm{AU}$ and $28200 \mathrm{AU}$, and $a_{\text {orig }_{25}}=29700 \mathrm{AU}$ and $22100 \mathrm{AU}$, for the TP and \#2 models, respectively. Hence the Oort spike is located at much closer orbits when stellar perturbations are at work. In addition, nearly all the comets in the spike are jumpers for the TP-model, while for Model \#2 almost all the comets in the lower range of semi-major axis are creepers or Kaib and Quinn creepers, and the jumpers are found mainly at semi-major axes larger than the maximum.

These differences can be explained by the TAZ filling shown in Fig. 6. We note that for semi-major axes larger than $13000 \mathrm{AU}$, the TAZ is much more filled in the TP-model than in any full model. In particular, the filling is at its maximum for semi-major axis around $50000 \mathrm{AU}$. This is exactly where the spike has its 


\section{Influence of the initial orbital energy distribution}

Let us now explore how the initial distribution of orbital energy affects our results concerning the current shape of the Oort cloud and flux of new comets. Our initial conditions are such that the quantity $z_{0}=-1 / a_{0}$ has a uniform distribution for $1,100<a_{0}<50000 \mathrm{AU}$, i.e., the density function of the initial orbital energy is constant. If we want a density function of this orbital energy proportional to $z_{0}^{\gamma}$, we just have to weigh each comet by

$$
w=C(\gamma+1)\left|z_{0}\right|^{\gamma}
$$




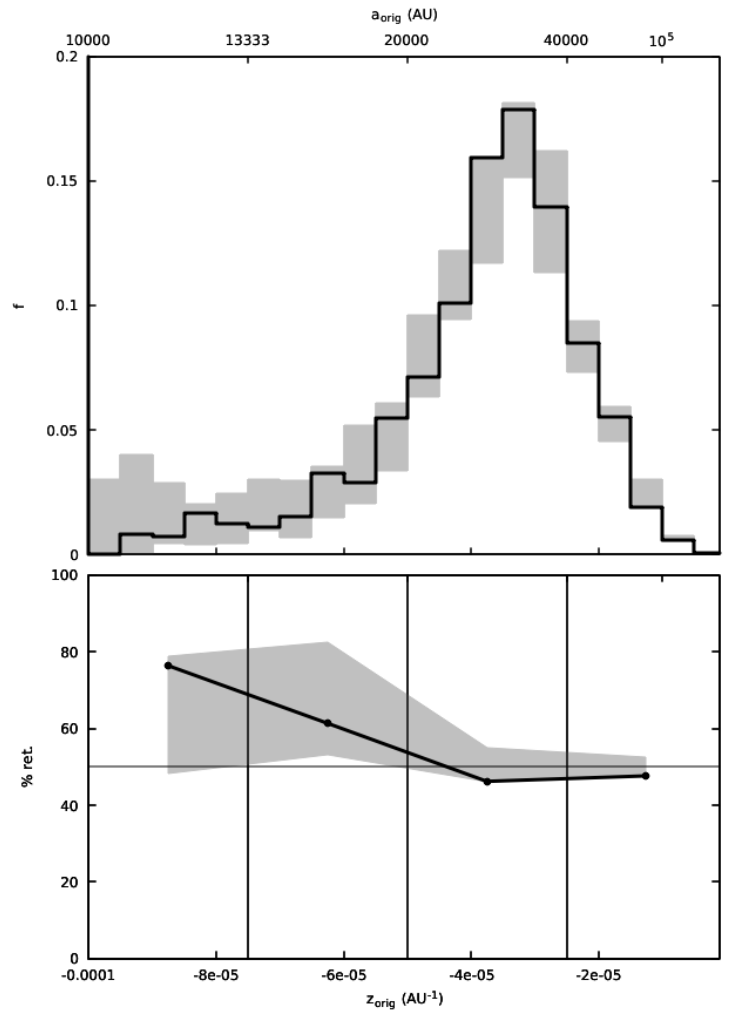

Figure 8: Same as Fig. 7 for Model \#2 only (black lines). The grey areas illustrate the ranges obtained using all the ten full models.

where

$$
C=\frac{z_{\max }-z_{\min }}{\left|z_{\min }\right|^{\gamma+1}-\left|z_{\max }\right|^{\gamma+1}}
$$

with $z_{\min }=-1100^{-1} \mathrm{AU}^{-1}$ and $z_{\max }=-50000^{-1} \mathrm{AU}^{-1}$.

We recall that, considering observable comets, we have to multiply this weight by $10^{6} / P_{\text {orig }}$ in order to get the equivalent number of observable comets per Myr, and that our initial conditions were defined by $\gamma=0$.

Figure 9 shows how the values of different current Oort cloud parameters depend on $\gamma$. The behaviour of all quantities except $a_{0_{50}}$ shows that our results are independent of $\gamma$, in the sense that the variations observed are always smaller than the ranges obtained from the ten stellar sequences. A key point here is shown by panel $b$ ) and $c$ ). As explained above, $\alpha$ and $\beta$ are the two indices describing the energy distribution of the current Oort cloud. The fact that these are not affected by the value of $\gamma$ means that the current Oort cloud is essentially independent of the distribution of orbital energy in the initial disk.

We have already seen that, during the time from $17 T_{G}$ to $21 T_{G}$, the Oort cloud has reached a quasisteady state, where the energy distribution is governed by planetary perturbations for $a<1000 \mathrm{AU}$ and 

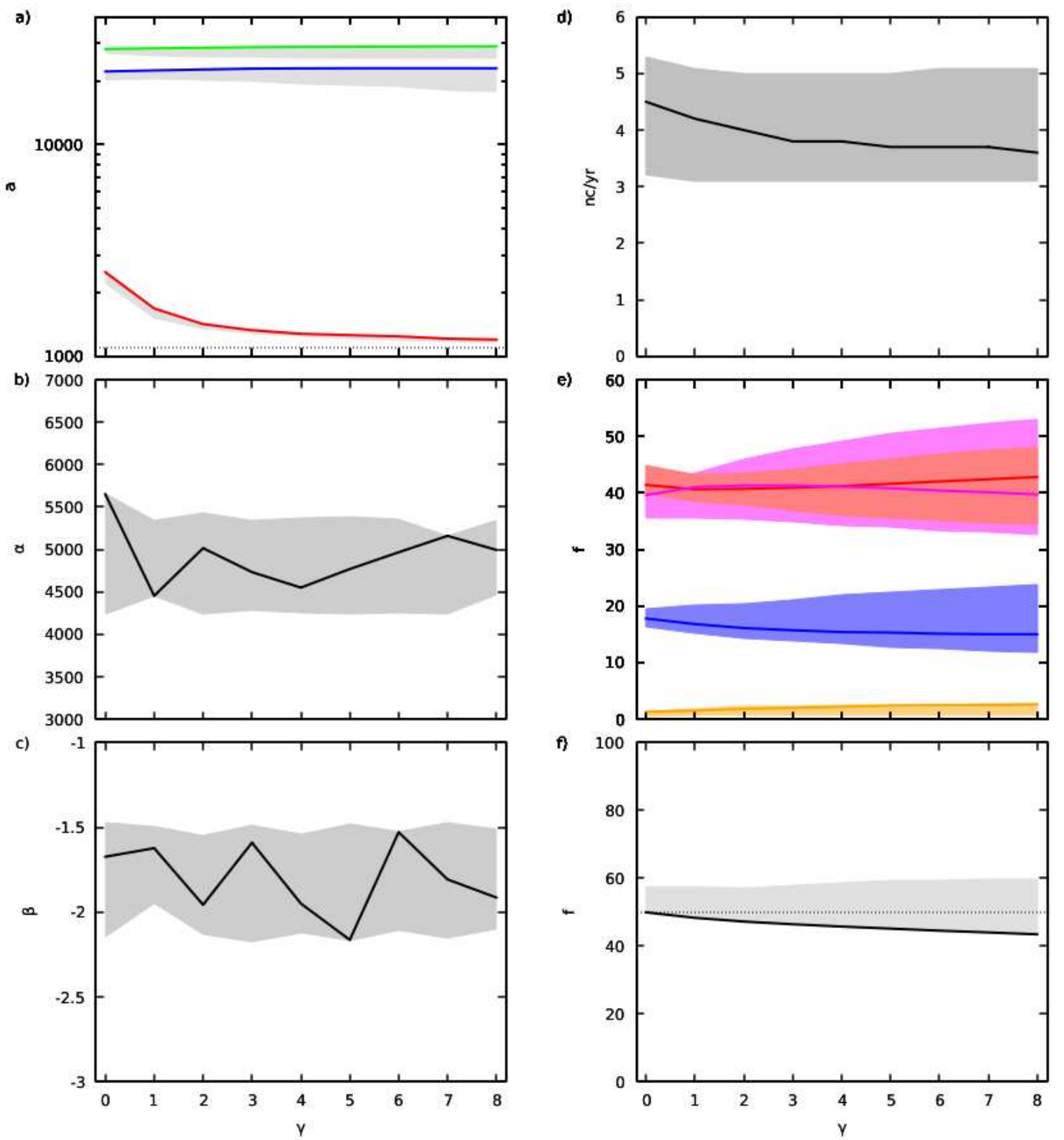

Figure 9: Variations of current Oort cloud parameters with respect to $\gamma \cdot a) a_{0_{50}}$ (red line), $a_{\mathrm{orig}_{25}}$ (blue line) and $a_{\text {orig }}$ (bo (green line); $b$ ) and $c$ ) the energy distribution parameters $\alpha$ and $\beta$, respectively; $d$ ) the number of observable comets per year; $e$ the fractions of the classes (jumpers in red, creepers in blue, KQ jumpers in orange and KQ creepers in magenta); and $f$ ) the fraction of retrograde orbits. In each case, the lines correspond to Model \#2 and the shaded or coloured areas indicate the range of results obtained from all ten stellar sequences.

by the Galactic tides and stellar encounters for $a>2000 \mathrm{AU}$. This state evolves only very slowly. Now we see that the structure of the current Oort cloud has lost memory of the initial distribution of orbital energy, from which it has evolved. 
A key point in understanding this feature is the fact that the initial perihelion is in the region of the planets and that the ecliptic inclination is small. As shown by Duncan et al. (1987), the energy transport caused by encounters with the planets then serves the role of feeding the comets into orbits that can be affected by the external agents, the tides and the stars. When this is achieved, it does not matter, in which initial orbit the comet was born.

The tidal regime is reached, when the strength of the tides is large enough to raise the perihelion out of the planetary influence. The faster is this transport of the perihelion by the tides, the better the comets will be protected from ejection by the planets. From this perspective, the Oort cloud can be viewed as a safe haven and storage facility, where the comets can stay for a time exceeding the age of the solar system. The role of stars has been reduced here to providing a transfer route in and out of the TAZ, thereby thermalizing the distribution. Quite naturally, this thermalization plays a crucial role in determining the flux of observable comets.

The orbital domain with $a<1000 \mathrm{AU}$, i.e., the planetary transport regime, is shaped by the planets. We cannot say that this region corresponds to the Oort cloud, since both tides and stars poorly affect the perihelion distance (see Fig 3 and Eq. 3). It is not the scope of the present paper to investigate the dynamics in this region, but we know from our simulations that our objects are far from being dynamically inert, since many comets (the decoupled one, for instance) transit through the region before going back to the Oort cloud.

Obviously, since we obtain the same kind of current Oort cloud with different initial conditions, as shown in panels $b$ ) and $c$ ) of Fig 9, we also obtain the same characteristics of the flux of observable comets, as shown in panels $d$ ) to $f$ ) of Fig 9. It is in fact a good surprise that even the flux of new comets is not strongly affected. This shows again the efficiency of the transport in orbital energy induced by the planets, since not only the structure but also the population of the current Oort cloud is rather insensitive to the initial orbital distribution.

Finally, the only quantity affected by the initial orbital energy distribution is obviously the median of the initial semi-major axis of the observable comets. This median converges to the lower bound of initial semi-major axis at $1100 \mathrm{AU}$ (shown by the dotted line in Fig. 9a). Again, this illustrates the independence of the final results on the initial orbital energy distribution. Clearly, the sample of comets that currently reach observable orbits is quite independent of the initial semi-major axis.

\section{Discussion}

From our simulation, two points should be discussed here. The first one is the definition of the Oort cloud, or in other words, what is an Oort cloud object? Generally, objects with perihelion distances large 
enough for their trajectories not to be affected by the planetary perturbations are considered to belong to the Oort cloud. Using such a criterion, Brasser and Morbidelli (2013) define a comet to be in the Oort cloud if both $a>1000 \mathrm{AU}$ and $q>40$ AU. From numerical simulation, Brasser and Schwamb (2015) are more conservative, saying that all objects with semi-major axis greater than $250 \mathrm{AU}$ and perihelion distance greater than $45 \mathrm{AU}$ belong to the Oort cloud. The problem of such definitions is that the Galactic tides may decrease the perihelion distance of an Oort cloud object so that it becomes a scattered disk object, just by an integrable process.

We propose here that an object is in the Oort cloud if, during a time span equal to the age of the solar system, the Galactic tides could raise the perihelion distance of the object so that its trajectory becomes unaffected by the perturbations of the giant planets.. More precisely, considering the conservative limiting value of $50 \mathrm{AU}$, we could say that an object belong to the Oort cloud, if the median effect of the tides is such that it can raise the perihelion distance to more than $50 \mathrm{AU}$ in less than 4.5 Gyr. This median effect is obtained considering the value of $q_{\text {med }}$ already used in Sec. 3.1 (see also Appendix A).

In Sec. 3.1 we had seen that, for a comet with a perihelion distance $q_{0}=32 \mathrm{AU}$, its semi-major axis has to be greater than $1090 \mathrm{AU}$ to be an Oort cloud comet, and for $q_{0}=15 \mathrm{AU}$, it has to be greater than 1640 AU. These values correspond rather well to the region where the transition between the planetary transport regime and the Galactic tide regime is located.

The second point to discuss is when does the Oort cloud reach its steady state? We know that this steady state is caused by the efficiency of the tides to raise the perihelion out of the planetary influence. Because the strength of the tides increases with the semi-major axis, it means that the greater is the semi-major axis, the sooner is reached the steady state. Then the question should be from which semi-major axis the steady-state is reached after a given time span? This corresponds to the shift from the transport regime imposed by the planets to the tidal regime. This shift is not easy to localize precisely. However, from Eq. A.4 in Appendix A, we see that the semi-major axis leading to a given change in perihelion distance during a time span $\Delta t$ decreases as $\sqrt{\Delta t}$. In other word, if one considers that the Oort cloud is in steady state for $a>2000 \mathrm{AU}$ after $4.5 \mathrm{Gyr}$, the semi-major axis $a_{s}$ beyond which it will be in steady state after a time span $\Delta t$ will be given by:

$$
a_{s}=2000 \sqrt{\frac{4.5}{\Delta t}} \mathrm{AU}
$$

where the unit ot time is the Gyr. Consequently after only 1 Gyr the Oort cloud has reached a steady state beyond $4240 \mathrm{AU}$, on the condition however that the Oort cloud was already populated in this region, that is the jovian planets had time to send comets in this region. 


\section{Conclusions}

In this paper we have introduced a new kind of Oort cloud model. It departs from an Oort cloud precursor in the form of a scattered disk with perihelia in the Uranus-Neptune region. The objects are launched into orbits with low inclinations and semi-major axes larger than $1100 \mathrm{AU}$. We trace their evolution over a time frame comparable with the age of the solar system, using passing stars, Galactic tides, and giant planets as perturbing agents. The orbital distributions in the cloud and the production of observable comets are analysed at five different epochs such that the position of the Sun in the Galaxy is always the same as the current one. We use ten different, randomly chosen stellar sequences and a very large $\left(10^{7}\right)$ sample of fictitious comets.

Our results show that the population of remaining objects reaches a steady state with a twofold shape: an inner part governed by planetary perturbation that only affect the orbital energy of the comets, and an outer one governed by the interaction between the Galactic tides and stars on one side and planetary perturbations on the other side. At the end of the integration time-span lasting about 4 billion years, the transition between this two parts was located at semi-major axis between 1000 and $2000 \mathrm{AU}$, and was at about $4000 \mathrm{AU}$ after an integration time-span lasting about one billion years. The outer part, which can be truly called the Oort cloud, converges quickly toward a Bolzmann distribution of the orbital energy.

As regards the flux of observable comets, we find that the role of stars with this setup to be quite different from the case, when the cloud is started in a thermalized state. In the latter situation, the TAZ gets depleted by ejections due to planets, but it is replenished by stellar perturbations. Hence, the stars help the production of observable comets in the long term, as found in earlier papers (Rickman et al., 2008; Fouchard et al., 2011a). However, our present initial conditions mean that the cloud is started with an overpopulation in the TAZ compared to the thermalized case. The stars then tend to thermalize the outer parts of the cloud, thereby decreasing the TAZ population and hampering the production of observable comets. This has drastic effects on the shape of the Oort spike. Indeed, because the jumpers mainly come from the region where the stars have depleted the TAZ, this class of observable comets has been strongly affected by the inclusion of stellar perturbation, reducing its importance and lowering the semi-major axis from which the jumpers come from. On the contrary, creepers and Kaib and Quinn creepers are much less affected by the inclusion of stellar perturbations since they come from regions closer to the Sun, in particular for the Kaib and Quinn creepers. It appears that between 55 and $60 \%$ of the observable comets are creepers or Kaib and Quinn creepers according to the stellar sequences considered. This is consistent with the results obtained by Dybczyński and Królikowska (2011). 
As we have been seen the stellar sequences do not affect significantly the shape of the Oort spike and the proportion of the different classes. On the contrary, the effect of the stellar sequences was more significant on both the total flux of observable comets (with a maximal flux being $60 \%$ larger than the minimal one) and the proportion of retrograde comets in the inner part of the Oort spike where almost all the observable comets are creepers or Kaib and Quinn creepers.

Then it has been highlighted that our results are independent on initial energy distribution of comets: shape of the Oort spike, shape of the final Oort cloud, strength of the final flux of observable comets. The only quantity significantly affected by the initial orbital distribution was the initial semi-major axis of observable comets: its median value is about $2300 \mathrm{AU}$ considering an uniform distribution of orbital energy, and quickly converges toward the lower bound of our initial condition, i.e. $1100 \mathrm{AU}$, when the initial distribution becomes steeper.

Our final conclusion is that, comparing Fouchard et al. (2014a) and the present study, one sees that the initial conditions strongly affect both the long term dynamics and the final structure. Consequently, a deep investigation of the observed Oort spike could give us some hint on the shape of the proto-Oort cloud and the mechanisms in action during the billion years of dynamical evolution of the cloud.

\section{Acknowledgements}

H.R. is indebted to Grant No. 2011/01/B/ST9/05442 of the Polish National Science Center. We gratefully acknowledge helpful remarks by the referees, Julio A. Fernández and Ramon Brasser.

\section{Appendix A. Evolution of the perihelion distance under an integrable Galactic tide}

Let us consider the dynamics under the action of only the normal component of the Galactic tides, i.e. the strongest one. The equations of motion thus obtained are then averaged over one orbital period of the comet, such that the dynamics of the mean orbital elements is completely integrable (Heisler and Tremaine, 1986). In this case, the evolution of the angular momentum $G=\sqrt{\mu^{\prime} a\left(1-e^{2}\right)}$, where $\mu^{\prime}=\mu M$ with $\mu$ the universal gravitational constant and $M$ the mass of the Sun and the giant planets, is given by:

$$
\left.\frac{\mathrm{d} G}{\mathrm{~d} t}=-\mathcal{G}_{3} \frac{5 L^{2}}{4 \mu^{\prime 2}} \sin 2 \omega_{G}\left(L^{2}-G^{2}\right)\right)\left(1-\frac{H^{2}}{G^{2}}\right),
$$

where $L=\sqrt{\mu^{\prime} a}$ and $H=G \cos i_{G}$, with $i_{G}$ and $\omega_{G}$ being the Galactic inclination and argument of perihelion, respectively, and $\mathcal{G}_{3}=4 \pi \mu \rho_{\odot}$ where $\rho_{\odot}$ is the local density of the Galactic disk.

When $q \ll a$, neglecting term in $(q / a)^{2}$, Eq. A.1 gives:

$$
\frac{\mathrm{d} \sqrt{q}}{\mathrm{~d} t}=\frac{5 \sqrt{2}}{8} \frac{\mathcal{G}_{3}}{\mu^{\prime}} \sin 2 \omega_{G} \sin ^{2} i_{G} a^{2} .
$$


For low semi-major axis and high eccentricity, the evolution of $\omega_{G}$ and $i_{G}$ is much smaller than the evolution of $q$ (see Matese and Whitman, 1992), consequently, one may consider than $\omega_{G}$ and $i_{G}$ are constant, at least for a time span significantly smaller than the period of the perihelion cycle induce by the tides (see Appendix B).

Hence, the maximal value $q_{\max }$ the perihelion can reach starting from $q_{0}$, during a time span equal at $\Delta T$ is given by:

$$
\sqrt{q_{\max }}-\sqrt{q_{0}} \approx \frac{5 \sqrt{2}}{8} \frac{\mathcal{G}_{3}}{\mu^{\prime}} a^{2} \Delta t .
$$

Instead of the maximal value, one can consider the median value reached by the perihelion distance by averaging the right hand side of Eq. A.2 over an uniform distribution of $\omega_{G}$ and $\cos i_{G}$. This yields:

$$
\sqrt{q_{\text {med }}}-\sqrt{q_{0}} \approx \frac{5 \sqrt{2}}{24} \frac{\mathcal{G}_{3}}{\mu^{\prime}} a^{2} \Delta t .
$$

\section{Appendix B. Period of perihelion distance cycle}

We consider again the same integrable system as in Appendix A. The formalism used by Breiter and Ratajczak (2005) will now be used. Let us first briefly recall their results.

In the integrable case we have, in addition to the mean Hamiltonian, two additional constants of motion:

$$
\alpha=\sqrt{1-e^{2}} \cos i_{G},
$$

and

$$
\beta=e^{2}\left(1-5 \sin ^{2} i_{G} \sin ^{2} \omega_{G}\right),
$$

where $e$ is the eccentricity, $i_{G}$ and $\omega_{G}$ are the Galactic inclination and argument of perihelion, respectively.

The sign of $\beta$ determines the kind of solution obtained: if $\beta<0$ then the argument of perihelion $\omega_{G}$ is librating, whereas if $\beta>0, \omega_{G}$ is circulating. If $\beta=0$ we obtained the homoclinic solution with an infinite period.

As in Breiter and Ratajczak (2005), let us define the following constants:

$$
\begin{aligned}
\gamma & =\frac{1}{4}\left(4-5 \alpha^{2}-\beta\right), \\
\kappa & =\sqrt{\gamma^{2}+\beta}, \\
\xi_{1} & =\frac{1}{2}(\gamma+\kappa), \\
\xi_{2} & =\frac{1}{2}(\gamma-\kappa) .
\end{aligned}
$$

Except for the homoclinic motion, the period of motion of the perihelion cycle is given by:

$$
T=\frac{1}{G \rho_{\odot} P} \frac{\mathbf{K}(m)}{\sqrt{\xi_{1}-\min \left(\beta, \xi_{2}\right)}}
$$


where $\mathbf{K}$ is the complete elliptic integral of the first kind, $P$ is the comet orbital period, $G$ the universal gravitational constant, $\rho_{\odot}$ the local density of the Galactic disk and $m$ is given by:

$$
m=\frac{\xi_{1}-\max \left(\beta, \xi_{2}\right)}{\xi_{1}-\min \left(\beta, \xi_{2}\right)} .
$$

The evolution of the eccentricity is such that its square value oscillates between a maximum given by $\xi_{1}$ and a minimum given by $\xi_{2}$ for the librating motion and $\beta$ for the circulating one.

For both the circulating and librating motion, the minimum of $T$ is obtained when $m=0$, that is when $\xi_{1}-\max \left(\beta, \xi_{2}\right)=0$. It this case $\mathbf{K}(0)=\pi / 2$.

Considering the librating case, one has $\beta<0$, and $\min \left(\beta, \xi_{2}\right)=\beta$. If $m=0$ then $\xi_{1}=\xi_{2}$, consequently $\beta=-\gamma^{2}$. We thus obtain:

$$
\xi_{1}-\min \left(\beta, \xi_{2}\right)=\xi_{1}-\beta=\frac{1}{2} \gamma+\gamma^{2},
$$

which is always smaller than 5 (and positive) since $\gamma \leq 2$.

This leads to the well known minimal possible value of the period of the eccentricity cycle equal to:

$$
P_{\min }=P_{\text {libr }}=\frac{\pi}{2 \sqrt{5} G \rho_{\odot} P} .
$$

However, this minimal value is obtained from the set of librating motion.

Let us now consider the case of circulating motions, i.e. $\beta>0$. Then the condition $m=0$ to have the minimal period gives $\xi_{1}=\beta$. By solving the equation and excluding the case $\beta=0$ (homoclinic motion), we get $\beta=\gamma+1 / 4$. Consequently

$$
\xi_{1}-\min \left(\beta, \xi_{2}\right)=\xi_{1}-\xi_{2}=\kappa=\gamma+\frac{1}{2} .
$$

which is always smaller than $5 / 2$. Then the minimal period of the eccentricity cycle considering only circulating motion is

$$
P_{\text {circ }}=\frac{\sqrt{2} \pi}{2 \sqrt{5} G \rho_{\odot} P}=\sqrt{2} P_{\text {libr }} .
$$

For our initial condition set, it appears than $65 \%$ of the comets correspond to librating solutions, whereas considering only the comets in the TAZ, given by the value of $\xi_{1}$, this proportion drops to $34 \%$. This difference is easily explicable from the expression for $\xi_{1}$. Consequently, from the expressions of the minimal period of the eccentricity obtained above, we see that this period is globally longer for the comets in the TAZ than for comets outside it. Indeed, it appears than globally the period of the eccentricity is about $20 \%$ higher for the TAZ comets than for all comets together.

R. Brasser, M. J. Duncan, H. F. Levison, Embedded star clusters and the formation of the Oort Cloud, Icarus 184 (2006) 59-82, doi:10.1016/j.icarus.2006.04.010. 
R. Brasser, M. J. Duncan, H. F. Levison, Embedded star clusters and the formation of the Oort Cloud II. The effect of the primordial solar nebula, Icarus 191 (2007) 413-433.

N. A. Kaib, T. Quinn, The formation of the Oort cloud in open cluster environments, Icarus 197 (2008) $221-238$.

G. Leto, M. Jakubík, T. Paulech, L. Neslušan, P. A. Dybczyński, The structure of the inner Oort cloud from the simulation of its formation for 2 Gyr, MNRAS 391 (2008) 1350-1358, doi:10.1111/j.1365-2966.2008.13975.x.

T. Paulech, M. Jakubík, L. Neslušan, P. A. Dybczyński, G. Leto, Probing the relation between the structure of initial protoplanetary disc and the Oort-cloud formation, AAP 509 A48, doi:10.1051/0004-6361/200912712.

H. Levison, M. Duncan, R. Brasser, D. Kaufmann, Capture of the Sun? Oort cloud from stars in its birth cluster, Science 329 (2010) 187-191.

R. Brasser, A. Higuchi, N. Kaib, Oort cloud formation at various Galactic distances, Astron. Astrophys. 516 (2010) A72-A84.

N. A. Kaib, R. Roškar, T. Quinn, Sedna and the Oort Cloud around a migrating Sun, Icarus 215 (2011) 491-507, doi: 10.1016/j.icarus.2011.07.037.

R. Brasser, A. Morbidelli, Oort cloud and Scattered Disc formation during a late dynamical instability in the Solar System, Icarus 225 (2013) 40-49.

A. Morbidelli, S. Marchi, W. F. Bottke, D. A. Kring, A sawtooth-like timeline for the first billion years of lunar bombardment, Earth and Planetary Science Letters 355 (2012) 144-151, doi:10.1016/j.epsl.2012.07.037.

H. Rickman, M. Fouchard, Ch. Froeschlé, G. B. Valsecchi, Injection of Oort Cloud comets: the fundamental role of stellar perturbations, Celestial Mechanics and Dynamical Astronomy 102 (2008) 111-132, doi:10.1007/s10569-008-9140-y.

M. Fouchard, Ch. Froeschlé, H. Rickman, G. B. Valsecchi, The key role of massive stars in Oort cloud comet dynamics, Icarus 214 (2011a) 334-347, doi:10.1016/j.icarus.2011.04.012.

M. Fouchard, H. Rickman, Ch. Froeschlé, G. B. Valsecchi, The last revolution of new comets: the role of stars and their detectability, AAP 535 A86, doi:10.1051/0004-6361/201116514.

N. A. Kaib, T. Quinn, Reassessing the Source of Long-Period Comets, Science 325 (2009) 1234-, doi:10.1126/science.1172676.

M. Fouchard, H. Rickman, Ch. Froeschlé, G. B. Valsecchi, Planetary perturbations for Oort Cloud comets. I. Distributions and dynamics, Icarus 222 (2013) 20-31, doi:10.1016/j.icarus.2012.10.027.

M. Duncan, T. Quinn, S. Tremaine, The formation and extent of the solar system comet cloud, AJ 94 (1987) 1330-1338, doi:10.1086/114571.

L. Dones, P. R. Weissman, H. F. Levison, M. J. Duncan, Oort cloud formation and dynamics, Comets II (2004) $153-174$.

H. F. Levison, M. J. Duncan, L. Dones, B. J. Gladman, The scattered disk as a source of Halley-type comets, Icarus 184 (2006) 619-633, doi:10.1016/j.icarus.2006.05.008.

H. F. Levison, L. Dones, M. J. Duncan, The Origin of Halley-Type Comets: Probing the Inner Oort Cloud, Astron. J 121 (2001) 2253-2267.

M. Fouchard, H. Rickman, Ch. Froeschlé, G. B. Valsecchi, Planetary perturbations for Oort cloud comets: II. Implications for the origin of observable comets, Icarus 231 (2014a) 110-121, doi:10.1016/j.icarus.2013.11.032.

P. Wiegert, S. Tremaine, The Evolution of Long-Period Comets, Icarus 137 (1999) 84-121.

M. Saillenfest, M. Fouchard, G. Tommei, G. B. Valsecchi, Study and application of the resonant secular dynamics beyond Neptune, Celest. Mech. Dynam. Astron. (2016) accepted.

R. Gomes, T. Gallardo, J. Fernández, A. Brunini, On The Origin of The High-Perihelion Scattered Disk: The Role of The Kozai Mechanism And Mean Motion Resonances., Celest. Mech. Dynam. Astron. 91 (2005) $109-129$.

J. Heisler, S. Tremaine, The influence of the galactic tidal field on the Oort comet cloud, Icarus 65 (1986) 13-26.

M. Fouchard, Ch. Froeschlé, H. Rickman, G. B. Valsecchi, Dynamical Features of the Oort Cloud Comets, in: J. Souchay, R. Dvorak (Eds.), Lecture Notes in Physics, Berlin Springer Verlag, vol. 790 of Lecture Notes in Physics, Berlin Springer Verlag, 401-430, 2010

A. Higuchi, E. Kokubo, H. Kinoshita, T. Mukai, Orbital Evolution of Planetesimals due to the Galactic Tide: Formation of the Comet Cloud, AJ 134 (2007) 1693-1706, doi:10.1086/521815.

R. Brasser, M. E. Schwamb, Re-assessing the formation of the inner Oort cloud in an embedded star cluster II. Probing the inner edge, MNRAS 446 (2015) 3788-3796.

T. Gallardo, G. Hugo, P. Pais, Survey of Kozai dynamics beyond Neptune, Icarus 220 (2012) 392-403, doi: 10.1016/j.icarus.2012.05.025.

J. H. Jeans, The Origin of Binary Systems, MNRAS 79 (1919) 408-416.

M. Fouchard, H. Rickman, Ch. Froeschlé, G. B. Valsecchi, Planetary perturbations for Oort cloud comets: III. Evolution of the cloud and production of centaurs and Halley type comets, Icarus 231 (2014b) 99-109, doi:10.1016/j.icarus.2013.11.034.

A. Higuchi, E. Kokubo, Effect of Stellar Encounters on Comet Cloud Formation, AJ 150 26, doi:10.1088/0004-6256/150/1/26.

S. Breiter, P. Dybczyński, A. Elipe, The action of the Galactic disk on the Oort cloud comets, A\&A 315 (1996) 618-624.

P. J. Francis, The Demographics of Long-Period Comets, APJ 635 (2005) 1348-1361, doi:10.1086/497684.

P. A. Dybczyński, M. Królikowska, Where do long-period comets come from? Moving through the Jupiter-Saturn barrier, MNRAS 416 (2011) 51-69, doi:10.1111/j.1365-2966.2011.19005.x.

J. J. Matese, P. G. Whitman, A model of the galactic tidal interaction with the Oort comet cloud, Celest. Mech. Dynam. Astron. 54 (1992) 13-35.

S. Breiter, R. Ratajczak, Vectorial elements for the Galactic disc tide effects in cometary motion, MNRAS 364 (2005) $1222-1228$. 